\title{
Erratum to: Mathematical properties of pump-leak models of cell volume control and electrolyte balance
}

\author{
Yoichiro Mori
}

Published online: 23 February 2012

(C) Springer-Verlag 2012

\begin{abstract}
Homeostatic control of cell volume and intracellular electrolyte content is a fundamental problem in physiology and is central to the functioning of epithelial systems. These physiological processes are modeled using pump-leak models, a system of differential algebraic equations that describes the balance of ions and water flowing across the cell membrane. Despite their widespread use, very little is known about their mathematical properties. Here, we establish analytical results on the existence and stability of steady states for a general class of pump-leak models. We treat two cases. When the ion channel currents have a linear current-voltage relationship, we show that there is at most one steady state, and that the steady state is globally asymptotically stable. If there are no steady states, the cell volume tends to infinity with time. When minimal assumptions are placed on the properties of ion channel currents, we show that there is an asymptotically stable steady state so long as the pump current is not too large. The key analytical tool is a free energy relation satisfied by a general class of pump-leak models, which can be used as a Lyapunov function to study stability.
\end{abstract}

Keywords Cell volume control · Electrolyte balance · Free energy · Lyapunov function · Differential algebraic system

Mathematics Subject Classification (2000) $\quad$ 92C30 $\cdot 34$ A09 $\cdot 34$ D20 $\cdot$ 34D23

Due to typographical errors inadvertently introduced into the orignal manuscript during the publisher's production process, the original publication is incorrect. The complete and correct version is given here.

The online version of the original article can be found under doi:10.1007/s00285-011-0483-8.

Y. Mori $(\bowtie)$

School of Mathematics, University of Minnesota, 206 Church St. SE, Minneapolis, MN 55455, USA

e-mail: ymori@umn.edu 


\section{Introduction}

Cells contain a large number of organic molecules that do not leak out through the cell membrane. The presence of organic molecules and their attendant counterions results in excess intracellular osmotic pressure. The plasma membrane is not mechanically strong enough to withstand significant differences in osmotic pressure, and thus the cell will tend to swell and burst. Plant cells and bacteria have a mechanically rigid cell wall to guard against this tendency. Animal cells maintain their cell volume with ionic pumps and ionic channels that together regulate the ionic composition of the cytosol (Hoffmann et al. 2009; Evans 2009; Boron and Boulpaep 2008).

This "pump-leak" mechanism is typically modeled in the following fashion (Keener and Sneyd 1998; Hoppensteadt and Peskin 2002). Consider a cell of volume $v$ and let $[\cdot]_{\mathrm{i}, \mathrm{e}}$ be the intracellular and extracellular ionic concentrations, respectively. We only consider the ions $\mathrm{Na}^{+}, \mathrm{K}^{+}$and $\mathrm{Cl}^{-}$. Let the cell be in a large and well-stirred extracellular bath so that $[\cdot]_{\mathrm{e}}$ can be assumed constant. We have the following balance equations for the three ionic species.

$$
\begin{aligned}
\frac{d}{d t}\left(F v\left[\mathrm{Na}^{+}\right]_{\mathrm{i}}\right) & =-g_{\mathrm{Na}}\left(\phi-\frac{R T}{F} \ln \left(\frac{\left[\mathrm{Na}^{+}\right]_{\mathrm{e}}}{\left[\mathrm{Na}^{+}\right]_{\mathrm{i}}}\right)\right)-3 \alpha F, \\
\frac{d}{d t}\left(F v\left[\mathrm{~K}^{+}\right]_{\mathrm{i}}\right) & =-g_{\mathrm{K}}\left(\phi-\frac{R T}{F} \ln \left(\frac{\left[\mathrm{K}^{+}\right]_{\mathrm{e}}}{\left[\mathrm{K}^{+}\right]_{\mathrm{i}}}\right)\right)+2 \alpha F, \\
\frac{d}{d t}\left(-F v\left[\mathrm{Cl}^{-}\right]_{\mathrm{i}}\right) & =-g_{\mathrm{Cl}}\left(\phi+\frac{R T}{F} \ln \left(\frac{\left[\mathrm{Cl}^{-}\right]_{\mathrm{e}}}{\left[\mathrm{Cl}^{-}\right]_{\mathrm{i}}}\right)\right) .
\end{aligned}
$$

Here, $\phi$ is the membrane potential, $g$. are the ion channel conductances for each species of ion, $\alpha$ is the strength of the pump current, $F$ is the Faraday constant, and $R T$ is the ideal gas constant times absolute temperature. The pump current for $\mathrm{Na}^{+}$and $\mathrm{K}^{+}$ has a ratio of 3:2 reflecting the stoichiometry of the Na-K ATPase (in Hoppensteadt and Peskin 2002, this ratio is set to 1:1 for simplicity). The above balance laws are supplemented by the following:

$$
\begin{aligned}
0= & {\left[\mathrm{Na}^{+}\right]_{\mathrm{i}}+\left[\mathrm{K}^{+}\right]_{\mathrm{i}}-\left[\mathrm{Cl}^{-}\right]_{\mathrm{i}}+\frac{z A}{v} } \\
= & {\left[\mathrm{Na}^{+}\right]_{\mathrm{e}}+\left[\mathrm{K}^{+}\right]_{\mathrm{e}}-\left[\mathrm{Cl}^{-}\right]_{\mathrm{e}}, } \\
\frac{d v}{d t}= & \zeta R T\left(\left[\mathrm{Na}^{+}\right]_{\mathrm{i}}+\left[\mathrm{K}^{+}\right]_{\mathrm{i}}+\left[\mathrm{Cl}^{-}\right]_{\mathrm{i}}+\frac{A}{v}\right. \\
& \left.-\left(\left[\mathrm{Na}^{+}\right]_{\mathrm{e}}+\left[\mathrm{K}^{+}\right]_{\mathrm{e}}+\left[\mathrm{Cl}^{-}\right]_{\mathrm{e}}\right)\right) .
\end{aligned}
$$

Equation (1.1d) is the electroneutrality condition, where $A$ is the total amount of organic molecules in the cell and $z$ the average charge on one organic molecule. We have assumed that there are no organic molecules outside the cell and that they do not pass through the membrane. Equation (1.1e) says that water flows into or out of the cell according to the osmotic pressure difference across the membrane. Here, $\zeta$ is the membrane permeability to water flow. System (1.1) forms a system of differential 
algebraic equations. We would like to see under what condition the above system possesses a stable steady state, representing a cell with a stable cell volume and ionic composition.

Models of this type were first introduced in Tosteson and Hoffman (1960), Tosteson (1964) and have since been extended and modified by several authors to study cell volume control (Jakobsson 1980; Lew et al. 1991; Hernández and Cristina 1998); (Armstrong 2003). Pump-leak models are widely used in epithelial physiology. Epithelial cells need to maintain their cell volume and ionic composition in the face of widely varying extracellular ionic and osmotic conditions. There is a large body of mathematical modeling work in the context of renal physiology (see Weinstein 1994, 2003 for review). Mathematical modeling studies of other systems using pump-leak models include Larsen et al. (2002), Fischbarg and Diecke (2005), and Yi et al. (2003).

Despite their widespread use and fundamental physiological importance, there seem to be very few analytical results regarding the behavior of pump-leak models. As for system (1.1), Keener and Sneyd (1998) show the following. Assuming $z \leq-1$, there is a unique steady state with a finite positive cell volume if and only if:

$$
\frac{\left[\mathrm{Na}^{+}\right]_{\mathrm{e}} \exp \left(-3 \alpha F /\left(g_{\mathrm{Na}} R T / F\right)\right)+\left[\mathrm{K}^{+}\right]_{\mathrm{e}} \exp \left(2 \alpha F /\left(g_{\mathrm{K}} R T / F\right)\right)}{\left[\mathrm{Na}^{+}\right]_{\mathrm{e}}+\left[\mathrm{K}^{+}\right]_{\mathrm{e}}}<1 .
$$

This means that if condition

$$
3\left[\mathrm{Na}^{+}\right]_{\mathrm{e}} / g_{\mathrm{Na}}>2\left[\mathrm{~K}^{+}\right]_{\mathrm{e}} / g_{\mathrm{K}}
$$

is satisfied, system (1.1) possesses a steady state for sufficiently small $\alpha>0$.

To the best of the author's knowledge, there are no analytical results on the stability of these steady states. In Weinstein (1997) the author studies an epithelial model of greater complexity than (1.1). The author obtains an algebraic expression for the linearized matrix around steady state and numerically studies its eigenvalues for physiological parameter values. The computation of this linearization is complicated by the presence of an algebraic constraint (the electroneutrality condition). Some authors have considered simpler non-electrolyte models of (epithelial) cell volume control and solute transport. Analytical results for such models can be found in Weinstein (1992), Hernández (2003, 2007), and Benson et al. (2010).

The goal of this paper is to establish analytical results on the existence and stability of steady states for a large class of pump-leak models that includes (1.1) and other representative models as special cases. In Sect. 2, we introduce the general class of pump-leak models that we shall treat in this paper. We consider $N$-species of ions subject to the electroneutrality constraint. Cell volume is controlled by the transmembrane osmotic pressure difference. The key observation of this Section is that the system of equations satisfies a free energy identity. This identity and its variations will be the main tool in studying the stability of steady states. The presence of a free energetic structure in pump-leak models leads us to natural structure conditions to be imposed on current-voltage relationships for ionic channel currents.

In Sect. 3, we study the case when the ionic channel current (or passive ionic flux) has a linear current-voltage relationship, the pump currents are constant and water flow is 
linearly proportional to the transmembrane osmotic pressure difference. System (1.1) is an example of such a system. We first establish the necessary and sufficient condition under which the system possesses a unique steady state. This condition, when applied to system (1.1), reduces to (1.2) (in fact, our conclusion is slightly stronger; we shall see that the restriction $z \leq-1$ is not needed in (1.2)). We then prove that this steady state is globally asymptotically stable. If a steady state does not exist, the cell volume $v$ tends to infinity as time $t \rightarrow \infty$ for any initial condition. The main tool in proving these statements is a modified version of the free energy introduced in Sect. 2. This modified free energy $\widetilde{G}$ satisfies $d \widetilde{G} / d t=-J, J \geq 0$, thus defining a Lyapunov function. Asymptotic stability of steady states follows by an examination of $\widetilde{G}$. To prove the global statements, we also make use of the fact that $J$ is a Lyapunov function. This is a consequence of the fact that, in a suitable set of variables, the system is a gradient flow of the convex function $\widetilde{G}$ with respect to a suitable metric. We thus have a clear dichotomy; if the system has a steady state, it is globally asymptotically stable and if not, the cell bursts. In Sect. 3.3, we discuss a simple epithelial model in which the cell is in contact with a mucosal and serosal bath. When the current voltage relationships for the ionic channels are all linear, the same stability results hold for this simple epithelial model.

In many modeling studies using the pump-leak model, the current-voltage relation for the ionic channel current is not linear. The popular Goldman current voltage relation is one such example. The goal of Sect. 4 is to establish a result on the existence and stability of steady states with minimal assumptions on the current-voltage relation. Indeed, all we assume here are properties required on thermodynamic grounds. We first discuss solvability of the differential algebraic system. Solvability is not entirely trivial given the algebraic constraint of electroneutrality. We then show that for a general class of pump-leak models, there is a steady state for sufficiently small positive pump rates so long as a generalization of condition (1.3) is satisfied. We then show that this steady state is asymptotically stable. Our main tool here is again the modified or relative free energy. The main difficulty in establishing stability is that the current voltage relation cannot in general be written as a function of the chemical potential jump only (this difficulty is not present when the current voltage relation is linear). We shall see that this effect is small when the pump rate is sufficiently small, which allows us to establish asymptotic stability of the steady state.

\section{Model formulation and the free energy identity}

Consider $N$ species of ion and let $c_{k}, k=1, \ldots, N$ be the intracellular concentrations of the $k$-th species of ion. We let $c_{k}^{\mathrm{e}}$ be the extracellular concentrations of these ions, which are assumed to be positive and constant independent of time. Let $v$ be the volume of the cell. The balance equation for the ions can be written as follows:

$$
\frac{d}{d t}\left(v c_{k}\right)=-j_{k}\left(\phi, \mathbf{c}, \mathbf{c}^{\mathrm{e}}\right)-p_{k}\left(\phi, \mathbf{c}, \mathbf{c}^{\mathrm{e}}\right), \quad k=1, \ldots, N .
$$

Here, we have written the transmembrane flux as the sum of the passive flux $j_{k}$ and the active flux $p_{k}$. The active flux, typically generated by ionic pumps, requires energy 
expenditure whereas the passive flux, carried by ionic channels and transporters, does not. The flux functions $j_{k}$ and $p_{k}$ depend on the transmembrane potential $\phi$ (intracellular potential minus the extracellular potential) as well as the vector of intracellular and extracellular concentrations $\mathbf{c}=\left(c_{1}, \ldots, c_{N}\right)^{T}$ and $\mathbf{c}^{\mathrm{e}}=\left(c_{1}^{\mathrm{e}}, \ldots, c_{N}^{\mathrm{e}}\right)^{T}$, where $\cdot^{T}$ denotes the transpose. We assume that $j_{k}$ and $p_{k}$ are $C^{1}$ functions of their arguments. Since the $\mathbf{c}^{\mathrm{e}}$ are assumed constant, the $\mathbf{c}^{\mathrm{e}}$ only appear as parameters of the above differential equation. The dependence on $\mathbf{c}^{\mathrm{e}}$ will thus often not be shown explicitly.

The functional form of the active flux function $p_{k}$ can be arbitrary, but for $j_{k}$, we must impose structure conditions so that it represents a passive flux. Commonly used examples of $j_{k}$ are:

$$
\begin{aligned}
j_{k}^{\mathrm{L}} & =G_{k}\left(R T \ln \left(\frac{c_{k}}{c_{k}^{\mathrm{e}}}\right)+F z_{k} \phi\right)=G_{k} \mu_{k}, \quad G_{k}>0, \\
j_{k}^{\mathrm{GHK}} & =P_{k} \frac{F z_{k} \phi}{R T} \frac{c_{k} \exp \left(\frac{F z_{k} \phi}{R T}\right)-c_{k}^{\mathrm{e}}}{\exp \left(\frac{F z_{k} \phi}{R T}\right)-1} \\
& =P_{k} c_{k}^{\mathrm{e}} \frac{F z_{k} \phi / R T}{\exp \left(\frac{F z_{k} \phi}{R T}\right)-1}\left(\exp \left(\frac{\mu_{k}}{R T}\right)-1\right), \quad P_{k}>0,
\end{aligned}
$$

where $R T$ is the ideal gas constant times absolute temperature, $F$ is the Faraday constant and $z_{k}$ is the valence of the $k$-th species of ion (e.g., 1 for $\mathrm{Na}^{+},-1$ for $\mathrm{Cl}^{-}$and so on). We assume that there is at least one ionic species for which $z_{k} \neq 0$. In the above, $\mu_{k}$ is the chemical potential of the $k$-th intracellular ion measured with respect to the extracellular bath:

$$
\mu_{k}=R T \ln \left(\frac{c_{k}}{c_{k}^{\mathrm{e}}}\right)+F z_{k} \phi
$$

Expression $j_{k}^{\mathrm{L}}$ is linear in $\mu_{k}$. If we multiply this by $F z_{k}$ to change units from ion flux into electric current, we obtain a linear current voltage relationship for this ionic current. This was used in (1.1). Expression $j_{k}^{\mathrm{GHK}}$ can be derived by assuming a constant electric field across the ion channel, and is known as the Goldman-Hodgkin-Katz current formula. An important feature of both $j_{k}^{\mathrm{L}}$ and $j_{k}^{\mathrm{GHK}}$ is that they are increasing functions of $\mu_{k}$ for fixed $\phi$ and that it is 0 when $\mu_{k}$ is 0 . In the general case, we require $j_{k}$ to satisfy a somewhat weaker version of these properties, which we shall discuss later in relation to Proposition 2.1.

Observe that $c_{k}$ can be expressed in terms of $\mu_{k}$ and $\phi$ through (2.4). We shall often find it useful to view $j_{k}$ as a function of $\phi$ and $\boldsymbol{\mu}=\left(\mu_{1}, \ldots, \mu_{N}\right)^{T}$ instead of $\phi$ and $\mathbf{c}$. We shall write this as $j_{k}(\phi, \mu)$ in a slight abuse of notation. We note that the dependence of $j_{k}$ on $\mu_{l}, l \neq k$ expresses the possibility that the flow of the $k$-th ion may be driven by the chemical potential gradient of the $l$-th ion. This is the case with many ionic transporters in which the flow of one species of ion is coupled to another. Indeed, many models of ionic transporter currents have this feature (Weinstein 1983; Strieter et al. 1990). 
Equation (2.1) is supplemented by:

$$
\begin{aligned}
0 & =\sum_{k=1}^{N} F z_{k} c_{k}+\frac{F z A}{v}=\sum_{k=1}^{N} F z_{k} c_{k}^{\mathrm{e}}, \\
\frac{d v}{d t} & =-j_{\mathrm{w}}\left(\mathbf{c}, \mathbf{c}^{\mathrm{e}}, v\right) .
\end{aligned}
$$

In (2.5), $A>0$ is the total amount of organic molecules inside the cell, and $z$ is the average valence of intracellular organic molecules. Equation (2.5) states that both the intracellular and extracellular concentrations satisfy the electroneutrality constraint. Electroneutrality of the extracellular space, together with the requirement that $z_{k} \neq 0$ for at least one $k$, requires that there must be at least two ionic species. In (2.6), $j_{\mathrm{w}}$ is the passive transmembrane water flux. An example of $j_{\mathrm{w}}$ is:

$$
j_{\mathrm{w}}=-\zeta \pi_{\mathrm{w}}, \pi_{\mathrm{w}}=R T\left(\sum_{k=1}^{N} c_{k}^{\mathrm{e}}-\left(\sum_{k=1}^{N} c_{k}+\frac{A}{v}\right)\right)
$$

where $\zeta>0$ is the hydraulic conductivity of water through the membrane. This simple prescription is what is used in (1.1) and many other models of cell volume and electrolyte control. In this example, water flow is proportional to $\pi_{\mathrm{w}}$, the osmotic pressure difference across the membrane, whose expression is given by the van't Hoff law. We shall assume that $j_{\mathrm{w}}$ is a $C^{1}$ function only of $\pi_{\mathrm{w}}$. The important property of (2.7) is that $j_{\mathrm{w}}=0$ when $\pi_{\mathrm{w}}=0$ and that it is increasing in $\pi_{\mathrm{w}}$. This property will be discussed further in relation to Proposition 2.1.

We seek solutions to the differential algebraic system (2.1), (2.5) and (2.6) given initial values for $\mathbf{c}=\left(c_{1}, \ldots, c_{N}\right)^{T}, v$ and $\phi$ that satisfy the algebraic constraint (2.5). We require that $c_{k}>0, k=1, \ldots, N$ and $v>0$ for all time. The membrane potential $\phi$ evolves so that the electroneutrality constraint (2.5) is satisfied at each instant. Multiplying (2.1) by $F z_{k}$ and summing in $k$, we have:

$$
I(\phi, \mathbf{c})=\sum_{k=1}^{N} F z_{k}\left(j_{k}(\phi, \mathbf{c})+p_{k}(\phi, \mathbf{c})\right)=0,
$$

where we used (2.5) to conclude that $I$, the total transmembrane electric current, must be 0 . This gives us an algebraic equation for $\phi$ that must be solved at each instant. The solvability of this equation is a necessary condition for the initial value problem to be solvable. For certain specific functional forms of $j_{k}$ and $p_{k}$, like those of (2.2) and (2.3) with $p_{k}$ constant, the solvability of (2.8) is immediate. We shall discuss the general case in Sect. 4.

One way to avoid the above difficulty arising from the algebraic constraint is to replace (2.5) with the following relation for intracellular concentrations:

$$
C_{\mathrm{m}} \phi=F\left(v \sum_{k=1}^{N} z_{k} c_{k}+z A\right)
$$


where $C_{\mathrm{m}}$ is the total membrane capacitance. This states that the total charge inside the cell is equal to the charge stored on the membrane capacitor. We note that (2.9) has its own difficulties as a biophysical model. If the concentrations are defined as the total amount of intracellular ions divided by volume, (2.9) is indeed correct. If we define $c_{k}$ to be the ionic concentration away from the surface charge layer (on the order of Debye length $\approx 1 \mathrm{~nm}$ in width), we must introduce surface ionic densities as was done in Mori and Peskin (2009). As we shall see shortly, the left hand side of (2.9) is often negligibly small and the electroneutrality condition (2.5) can thus be seen as a perturbative limit of condition (2.9). We shall discuss this point further after we make our system dimensionless.

Scale ionic concentration, volume and membrane potential as follows and introduce the primed dimensionless variables:

$$
c_{k}=c_{0} c_{k}^{\prime}, c_{k}^{\mathrm{e}}=c_{0} c_{k}^{\mathrm{e} \prime}, v=v_{0} v^{\prime}, \phi=\frac{R T}{F} \phi^{\prime},
$$

where $c_{0}$ and $v_{0}$ are the typical concentrations and volumes, respectively. Equations (2.1), (2.5) and (2.6) become:

$$
\begin{aligned}
\frac{d\left(v^{\prime} c_{k}^{\prime}\right)}{d t^{\prime}} & =-j_{k}^{\prime}\left(\phi^{\prime}, \boldsymbol{\mu}^{\prime}\right)-p_{k}^{\prime}\left(\phi^{\prime}, \mathbf{c}^{\prime}\right), \quad \mu_{k}^{\prime}=\ln \left(\frac{c_{k}^{\prime}}{c_{k}^{\mathrm{e} \prime}}\right)+z_{k} \phi^{\prime}, \\
0 & =\sum_{k=1}^{N} z_{k} c_{k}^{\prime}+z \frac{A^{\prime}}{v^{\prime}}=\sum_{k=1}^{N} z_{k} c_{k}^{\mathrm{e} \prime}, \quad A^{\prime}=\frac{A}{c_{0} v_{0}}, \\
\frac{d v^{\prime}}{d t^{\prime}} & =-j_{\mathrm{w}}^{\prime}\left(\pi_{\mathrm{w}}^{\prime}\right), \quad \pi_{\mathrm{w}}^{\prime}=\sum_{k=1}^{N} c_{k}^{\mathrm{e} \prime}-\left(\sum_{k=1}^{N} c_{k}^{\prime}+\frac{A^{\prime}}{v^{\prime}}\right),
\end{aligned}
$$

where $\boldsymbol{\mu}^{\prime}=\left(\mu_{1}^{\prime}, \ldots, \mu_{N}^{\prime}\right)^{T}$ and $\mathbf{c}^{\prime}=\left(c_{1}^{\prime}, \ldots, c_{N}^{\prime}\right)^{T}$. Time $t$, the flux functions $j_{k}, p_{k}$ and $j_{\mathrm{w}}$ are suitably rescaled to yield their respective primed variables. We note that it is possible to further reduce the number of constants, for example, by taking $c_{0}$ to be the total extracellular concentration. We shall not pursue this here, since it leads to some difficulty in understanding the physical meaning of each term in the resulting dimensionless system.

Equation (2.9) yields:

$$
\epsilon \phi^{\prime}=\left(v^{\prime} \sum_{k=1}^{N} z_{k} c_{k}^{\prime}+z A^{\prime}\right), \quad \epsilon=\frac{C_{\mathrm{m}} R T / F}{F c_{0} v_{0}}
$$

where $\epsilon$ is a dimensionless parameter expressing the ratio between the amount of ions contributing to the surface charge and the absolute amount of charge in the cytosolic bulk. This quantity is typically very small (about $10^{-7}$ ) and we thus expect that it is an excellent approximation to let the left hand side of (2.12) be 0 and adopt condition (2.5) (or its dimensionless version (2.11b)), if the membrane potential does not vary too rapidly. Most, if not all modeling studies of cellular electrolyte and water balance use the electroneutrality constraint (2.5) or (2.11b) and we shall treat this case only. 
We shall henceforth deal almost exclusively with the dimensionless system. To avoid cluttered notation, we remove the primes from the dimensionless variables.

An important property of the above system is that it possesses a natural energy function.

Proposition 2.1 Let $v$ and $c_{k}, k=1, \ldots, N$ satisfy system (2.11). Then, the following equality holds:

$$
\frac{d G}{d t}=-\sum_{k=1}^{N} \mu_{k}\left(j_{k}+p_{k}\right)-\pi_{\mathrm{w}} j_{\mathrm{w}}
$$

where

$$
G=v \sigma, \quad \sigma=\sum_{k=1}^{N}\left(c_{k}\left(\ln \left(\frac{c_{k}}{c_{k}^{\mathrm{e}}}\right)-1\right)+c_{k}^{\mathrm{e}}\right)+\frac{A}{v}\left(\ln \left(\frac{A}{v}\right)-1\right)
$$

Identity (2.13) is not entirely new. In Sauer (1973), Fromter (1974), and Weinstein (1983) the authors argue on thermodynamic grounds that free energy dissipation and input for an epithelial system should be given by the right hand side of (2.13). The new observation here is that, by appropriately defining a free energy function (that is to say, the left hand side of (2.13)), this thermodynamic property can be turned into a mathematical statement about system (2.11). We also point out that a similar identity valid for a system of partial differential equations describing electrodiffusion and osmosis was proved in Mori et al. (2011). In subsequent sections we will use this as a tool to study stability of states.

Proof of Proposition 2.1 View $\sigma$ as a function of $c_{k}$ and $c_{A}=A / v$. Note that:

$$
\frac{d}{d t}\left(v c_{A}\right)=\frac{d A}{d t}=0
$$

since $A$ is constant. Define the chemical potential of intracellular organic molecules as:

$$
\mu_{A}=\ln c_{A}+z \phi=\frac{\partial \sigma}{\partial c_{A}}+z \phi
$$

Note that:

$$
\mu_{k}=\frac{\partial \sigma}{\partial c_{k}}+z_{k} \phi
$$


Multiply (2.1) by $\mu_{k}$, multiply (2.15) by $\mu_{A}$ and take the sum. The left hand side yields:

$$
\begin{aligned}
& \sum_{k=1}^{N} \mu_{k} \frac{d}{d t}\left(v c_{k}\right)+\mu_{A} \frac{d}{d t}\left(v c_{A}\right) \\
& \quad=\sum_{k=1}^{N}\left(\frac{\partial \sigma}{\partial c_{k}}+z_{k} \phi\right) \frac{d}{d t}\left(v c_{k}\right)+\left(\frac{\partial \sigma}{\partial c_{A}}+z \phi\right) \frac{d}{d t}\left(v c_{A}\right) \\
& =\frac{d}{d t}(v \sigma)+\left(\sum_{k=1}^{N} c_{k} \frac{\partial \sigma}{\partial c_{k}}+c_{A} \frac{\partial \sigma}{\partial c_{A}}-\sigma\right) \frac{d v}{d t}+\phi \frac{d}{d t}\left(v \sum_{k=1}^{N} z_{k} c_{k}+z v c_{A}\right) \\
& \quad=\frac{d}{d t}(v \sigma)-\pi_{\mathrm{w}} \frac{d v}{d t}
\end{aligned}
$$

where we used (2.5) and (2.14) in the third equality. We thus have:

$$
\frac{d}{d t}(v \sigma)-\pi_{\mathrm{w}} \frac{d v}{d t}=-\sum_{k=1}^{N} \mu_{k}\left(j_{k}+p_{k}\right) .
$$

Equation (2.13) thus follows from (2.6).

The function $\sigma$ should be interpreted as the free energy per unit volume of intracellular electrolyte solution. A key fact that was used in the above proof is the identity:

$$
\pi_{\mathrm{w}}=\sigma-\left(\sum_{k=1}^{N} c_{k} \frac{\partial \sigma}{\partial c_{k}}+c_{A} \frac{\partial \sigma}{\partial c_{A}}\right) .
$$

This relation, connecting the free energy with osmotic pressure, is well-known in physical chemistry (Doi and See 1996).

When $p_{k}=0, k=1, \ldots, N$ in (2.13), there are no active currents and we have:

$$
\frac{d G}{d t}=-\sum_{k=1}^{N} \mu_{k} j_{k}-\pi_{\mathrm{w}} j_{\mathrm{w}}
$$

Given the interpretation of $G$ as the total free energy of the system, the second law of thermodynamics requires that $G$ be decreasing in time. The negativity of (2.21) when $\boldsymbol{\mu} \neq \mathbf{0}$ and $\pi_{\mathrm{w}} \neq 0$ is equivalent to the statement that the following conditions be satisfied:

$$
\begin{array}{cl}
\sum_{k=1}^{N} \mu_{k} j_{k}(\phi, \mu)>0 & \text { for all } \phi \text { and } \boldsymbol{\mu} \neq \mathbf{0} \\
\pi_{\mathrm{w}} j_{\mathrm{w}}\left(\pi_{\mathrm{w}}\right)>0 & \text { if } \pi_{\mathrm{w}} \neq 0 .
\end{array}
$$


Condition (2.22), together with continuity of $j_{k}$ and $j_{\mathrm{w}}$ with respect to its arguments immediately implies that:

$$
j_{k}(\phi, \mu=\mathbf{0})=0, \quad k=1, \ldots, N, \quad j_{\mathrm{w}}\left(\pi_{\mathrm{w}}=0\right)=0,
$$

where the conditions on $j_{k}$ is to be satisfied for all $\phi$. Taking the derivative of the above expression for $j_{k}$ with respect to $\phi$, we see that:

$$
\frac{\partial j_{k}}{\partial \phi}(\phi, \boldsymbol{\mu}=\mathbf{0})=0, \quad k=1, \ldots, N
$$

We shall find this expression useful later on.

Let us require that the derivative of $j_{\mathrm{w}}$ with respect to $\pi_{\mathrm{w}}$ be non-zero at $\pi_{\mathrm{w}}=0$. This non-degeneracy condition, together with (2.23), leads to:

$$
\frac{\partial j_{\mathrm{w}}}{\partial \pi_{\mathrm{w}}}\left(\pi_{\mathrm{w}}=0\right)>0 \text {. }
$$

Let $\mathbf{j}=\left(j_{1}, \ldots, j_{N}\right)^{T}$ and let $\partial \mathbf{j} / \partial \boldsymbol{\mu}$ be the Jacobian matrix with respect to $\mu$ for fixed $\phi$. That is to say, the $k l$ entry of the $N \times N$ matrix $\partial \mathbf{j} / \partial \boldsymbol{\mu}$ is given by $\partial j_{k} / \partial \mu_{l}$. The non-degeneracy condition for $\mathbf{j}$ is that $\partial \mathbf{j} / \partial \boldsymbol{\mu}$ be non-singular at $\boldsymbol{\mu}=0$. We require the following condition:

$$
\frac{\partial \mathbf{j}}{\partial \boldsymbol{\mu}}(\phi, \boldsymbol{\mu}=\mathbf{0}) \text { is symmetric positive definite for all } \phi .
$$

The symmetry of the Jacobian matrix does not follow from the non-degeneracy condition and condition (2.22). In fact, these two conditions imply only that:

$$
\frac{1}{2}\left(\frac{\partial \mathbf{j}}{\partial \boldsymbol{\mu}}+\left(\frac{\partial \mathbf{j}}{\partial \boldsymbol{\mu}}\right)^{T}\right)(\phi, \boldsymbol{\mu}=\mathbf{0}) \text { is symmetric positive definite for all } \phi .
$$

The symmetry of $\partial \mathbf{j} / \partial \boldsymbol{\mu}$ is required by the Onsager reciprocity principle (Onsager 1931; Katzir-Katchalsky and Curran 1965; Kjelstrup and Bedeaux 2008). We note that (2.27) is the same as the condition introduced in Sauer (1973), Fromter (1974), and Weinstein (1983). It is easy to see that both (2.2) and (2.3) satisfy (2.22), (2.24) and (2.27) and that (2.7) satisfies (2.23), (2.24) and (2.26).

Note that (2.23) together with (2.11c) implies that the intracellular and extracellular osmotic pressures must be equal at steady state. We are thus assuming that the membrane cannot generate any mechanical force to balance a difference in osmotic pressure. If the cell membrane (or its attendant structures) can generate some elastic force, it would make it easier for the cell to maintain its volume.

Our starting point in deriving the above structure conditions for $j_{k}$ and $j_{\mathrm{w}}$ was the requirement that the right hand side of (2.21) be negative. If $j_{k}$ is allowed to depend on $\pi_{\mathrm{w}}$ and $j_{\mathrm{w}}$ on $\boldsymbol{\mu}$, a more general structure condition can be formulated. Although 
it should not be difficult to extend the results to follow to this more general case, we will not pursue this here to keep the presentation reasonably simple.

\section{Results when the flux functions are linear in the chemical potential jump}

Before dealing with the general case in Sect. 4, we treat the simpler case when $j_{k}$ and $j_{\mathrm{w}}$ are linear in $\boldsymbol{\mu}$ and $\pi_{\mathrm{w}}$, respectively, and $p_{k}$ are constants in (2.11). In this case, we obtain a more or less complete picture of the behavior of our system. System (2.11) becomes:

$$
\begin{aligned}
\frac{d}{d t}(v \mathbf{c}) & =-L \boldsymbol{\mu}-\mathbf{p}, \\
0 & =\sum_{k=1}^{N} z_{k} c_{k}+\frac{z A}{v}=\sum_{k=1}^{N} z_{k} c_{k}^{\mathrm{e}}, \\
\frac{d v}{d t} & =-\zeta \pi_{\mathrm{w}}
\end{aligned}
$$

where $L=\partial \mathbf{j} / \partial \boldsymbol{\mu}$ is an $N \times N$ matrix, which by (2.27), is symmetric positive definite, and $\mathbf{p}=\left(p_{1}, \ldots, p_{N}\right)^{T}$ is the vector of the active fluxes. The hydraulic permeability $\zeta=\partial j_{\mathrm{w}} / \partial \pi_{\mathrm{w}}$ is positive by (2.26). The extracellular ionic concentrations $c_{k}^{\mathrm{e}}>0, k=1, \ldots, N$ and the amount of impermeable organic solute $A$ are assumed positive as discussed in the previous Section. We seek solutions $(\mathbf{c}, v) \in \mathbb{R}_{+}^{N} \times \mathbb{R}_{+}$ where $\mathbb{R}_{+}$denotes the set of positive real numbers.

Here and in the sequel, we shall often find it useful to refer to the pair $(\mathbf{c}, v)$ as well as the triple $(\mathbf{c}, v, \phi) \in \mathbb{R}_{+}^{N} \times \mathbb{R}_{+} \times \mathbb{R}$. We shall often view the pair and the triple as being members of $\mathbb{R}_{+}^{N+1}$ and $\mathbb{R}_{+}^{N+1} \times \mathbb{R}$, respectively, and write (c, $\left.v\right) \in$ $\mathbb{R}_{+}^{N+1}$ and $(\mathbf{c}, v, \phi) \in \mathbb{R}_{+}^{N+1} \times \mathbb{R} \subset \mathbb{R}^{N+2}$. The more "correct" notation may be to write $\left(\mathbf{c}^{T}, v\right)^{T}=\left(c_{1}, \ldots, c_{N}, v\right)^{T} \in \mathbb{R}_{+}^{N+1}, \quad\left(\mathbf{c}^{T}, v, \phi\right)^{T}=\left(c_{1}, \ldots, c_{N}, v, \phi\right)^{T} \in$ $\mathbb{R}_{+}^{N+1} \times \mathbb{R}$ given that $\mathbf{c}$ is a column vector. We will not adopt this unnecessarily ugly notation. Similar comments apply to the pair $(\mathbf{a}, v)$ and the triple $(\mathbf{a}, v, \phi)$ where $\mathbf{a}=v \mathbf{c}$.

For system (3.1), we may compute $\phi$ explicitly in terms of $\mathbf{c}$ by solving the (dimensionless version of) (2.8):

$$
\phi=-\frac{\langle\mathbf{z}, L \boldsymbol{\gamma}+\mathbf{p}\rangle_{\mathbb{R}^{N}}}{\langle\mathbf{z}, L \mathbf{z}\rangle_{\mathbb{R}^{N}}}, \quad \boldsymbol{\gamma}=\left(\gamma_{1}, \ldots, \gamma_{N}\right)^{T}, \gamma_{k}=\ln \left(\frac{c_{k}}{c_{k}^{\mathrm{e}}}\right)
$$

where $\mathbf{z}=\left(z_{1}, \ldots, z_{N}\right)^{T}$. Substituting the above expression for $\phi$ into (3.1a), we obtain:

$$
\begin{aligned}
\frac{d}{d t}(v \mathbf{c}) & =-\widehat{L}(\boldsymbol{\gamma}+\mathbf{q}), \quad \widehat{L}_{k l}=L_{k l}-\frac{(L \mathbf{z})_{k}(L \mathbf{z})_{l}}{\langle\mathbf{z}, L \mathbf{z}\rangle_{\mathbb{R}^{N}}} \\
\mathbf{q} & =\left(q_{1}, \ldots, q_{N}\right)^{T}, \quad \mathbf{q}=L^{-1} \mathbf{p}
\end{aligned}
$$


where $L_{k l}, \widehat{L}_{k l}$ are the $k l$ entries of the $N \times N$ matrix $L, \widehat{L}$ and $(L \mathbf{z})_{k}$ is the $k$-th component of the vector $L \mathbf{z} \in \mathbb{R}^{N}$. Note that $L^{-1}$ exists given that $L$ is positive definite. Replacing (3.1a) with (3.3), we now have a system of ordinary differential equations (ODEs) for $\mathbf{c}$ and $v$ only. Applying the standard existence and uniqueness theorem for ODEs, we conclude that a unique solution always exist for sufficiently short time so long as the solution remains in (c, $v) \in \mathbb{R}_{+}^{N+1}$.

Even though our ODE system is for $N+1$ variables $\mathbf{c}=\left(c_{1}, \ldots, c_{N}\right)^{T}$ and $v$, our dynamical system is only $N$ dimensional, since the dynamics is constrained by the electroneutrality condition (3.1b). The initial value problem for (3.1) can thus only be solved if the initial values satisfy (3.1b). In the proof of Proposition 3.4, we will find it useful to make a change of variables to remove this constraint.

3.1 Existence of steady states and asymptotic stability

Our first observation is the following.

Proposition 3.1 Consider the function:

$$
f(\phi)=\sum_{k=1}^{N} c_{k}^{\mathrm{e}}\left(\exp \left(-q_{k}-z_{k} \phi\right)-1\right),
$$

where $q_{k}, k=1, \ldots, N$ was defined in (3.3). The function $f(\phi), \phi \in \mathbb{R}$ has a unique minimizer $\phi=\phi_{\min }$. System (3.1) has a unique steady state if this minimum value is negative:

$$
f_{\min }\left(\mathbf{q}, \mathbf{c}^{\mathrm{e}}, \mathbf{z}\right) \equiv f\left(\phi_{\min }\right)<0 .
$$

Otherwise, the system does not have any steady states.

The above condition can be interpreted as follows. At steady state, it is easily seen that the concentrations $c_{k}$ must be equal to $c_{k}^{*}=c_{k}^{\mathrm{e}} \exp \left(-q_{k}-z_{k} \phi^{*}\right)$ where $\phi^{*}$ is the value of $\phi$ at steady state (see (3.7)). We need $f\left(\phi^{*}\right)=\sum_{k=1}^{N}\left(c_{k}^{*}-c_{k}^{\mathrm{e}}\right)<0$ since there must be "osmotic room" for the impermeable solutes. This is only possible if the minimum of $f(\phi), \phi \in \mathbb{R}$ is negative. We also point out that the above condition depends only on $\mathbf{q}=L^{-1} \mathbf{p}, \mathbf{c}^{\mathrm{e}}$ and $\mathbf{z}$ and does not depend on $z$ or $A$.

Proof of Proposition 3.1 Set the right hand side of (3.1a) and (3.1c) to zero. We have:

$$
\mathbf{q}=L^{-1} \mathbf{p}=-\boldsymbol{\mu}, \quad \pi_{\mathrm{w}}=0 .
$$

Solving for $c_{k}$ in the first expression we have:

$$
c_{k}=c_{k}^{\mathrm{e}} \exp \left(-q_{k}-z_{k} \phi\right), \quad k=1 \ldots, N .
$$


Substitute this into $\pi_{\mathrm{w}}=0$ and (3.1b). We have:

$$
\begin{aligned}
f(\phi)+\frac{A}{v} & =0 \\
-\frac{d f}{d \phi}+\frac{z A}{v} & =\sum_{k=1}^{N} z_{k} c_{k}^{\mathrm{e}} \exp \left(-q_{k}-z_{k} \phi\right)+\frac{z A}{v}=0,
\end{aligned}
$$

where $f(\phi)$ is given by (3.4). We must find solutions $\phi$ and $v>0$ to the above system. Note that:

$$
\frac{d^{2} f}{d \phi^{2}}=\sum_{k=1}^{N} z_{k}^{2} c_{k}^{\mathrm{e}} \exp \left(-q_{k}-z_{k} \phi\right)>0, \quad \lim _{\phi \pm \infty} \frac{d f}{d \phi}= \pm \infty
$$

The second property comes from the fact that there are ions with negative and positive valences among the $N$ species of ions and that $c_{k}^{\mathrm{e}}>0$. We can thus solve (3.9) for $\phi$ uniquely in terms of $v$. Let this function be $\phi=\varphi(v)$. We have:

$$
\frac{d \varphi}{d v}=-\left(\frac{d^{2} f}{d \phi^{2}}\right)^{-1} \frac{z A}{v^{2}}
$$

Consider the left hand side of (3.8) and substitute $\phi=\varphi(v)$ into this expression:

$$
R(v) \equiv f(\varphi(v))+\frac{A}{v}=0 .
$$

Our problem of finding steady states is reduced to the question of whether the above equation in $v$ has a positive solution. We have:

$$
\frac{d R}{d v}=\frac{d f}{d \phi} \frac{d \varphi}{d v}-\frac{A}{v^{2}}=-\left(\frac{d^{2} f}{d \phi^{2}}\right)^{-1} \frac{(z A)^{2}}{v^{3}}-\frac{A}{v^{2}} \leq-\frac{A}{v^{2}}<0
$$

where we used (3.9) and (3.11) in the second equality and (3.10) in the first inequality. Therefore, $R(v)$ is monotone decreasing. Note that:

$$
R(\epsilon)=R(1)-\int_{\epsilon}^{1}\left(\frac{d R}{d v}\right) d v \geq R(1)+\int_{\epsilon}^{1}\left(\frac{A}{v^{2}}\right) d v=R(1)+A\left(\epsilon^{-1}-1\right) .
$$

Therefore, $R(v) \rightarrow \infty$ as $v$ tends to 0 from above. Thus, (3.12) has a unique positive solution if:

$$
\lim _{v \rightarrow \infty} R(v)<0,
$$


and otherwise, there is no solution. Let $\varphi_{\infty}=\lim _{v \rightarrow \infty} \varphi(v)$. Note that this limit exists since, by (3.11), $\varphi(v)$ is monotone if $z \neq 0$ and constant if $z=0$. Taking the limit $v \rightarrow \infty$ on both sides of (3.9), we see that $\varphi_{\infty}$ is the unique solution to $d f / d \phi=0$ as an equation for $\phi$. Given (3.12), condition (3.15) can be written as $f\left(\varphi_{\infty}\right)<0$. The statement follows by taking $\phi_{\min }=\varphi_{\infty}$.

Condition (3.5) applied to (1.1) yields condition (1.2). Since condition (3.5) is valid regardless of the value of $z$, we may lift the restriction $z \leq-1$ found in Keener and Sneyd (1998).

Fix $\mathbf{q}=L^{-1} \mathbf{p}$ and $\mathbf{c}^{\mathrm{e}}$ so that (3.5) is satisfied. Since condition (3.5) does not depend on $z$ or $A$, a unique steady state $(\mathbf{c}, v, \phi)=\left(\mathbf{c}^{*}, v^{*}, \phi^{*}\right)$ exists for any value of $z$ and $A>0$. We may thus view $\left(\mathbf{c}^{*}, v^{*}, \phi^{*}\right)$ as a function of $A$ and $Q_{A}=z A$, defined for $A>0$ and $Q_{A} \in \mathbb{R}$. We can compute the dependence of $v^{*}$ on $Q_{A}$ and $A$ as follows.

$$
\begin{aligned}
\frac{\partial v^{*}}{\partial A} & =\left(\left(\frac{Q_{A}}{v^{*}}\right)^{2}+\frac{d^{2} f}{d \phi^{2}} \frac{A}{v^{*}}\right)^{-1} \frac{d^{2} f}{d \phi^{2}}>0, \\
\frac{\partial v^{*}}{\partial Q_{A}} & =\left(\left(\frac{Q_{A}}{v^{*}}\right)^{2}+\frac{d^{2} f}{d \phi^{2}} \frac{A}{v^{*}}\right)^{-1} \frac{Q_{A}}{v^{*}} .
\end{aligned}
$$

From a biophysical standpoint, (3.16) is reasonable since more impermeable solute leads to greater osmotic pressure. Note that (3.17) says that the $v^{*}$ increases if the absolute amount of charge (whether negative or positive) increases. This is also biophysically reasonable since more charge on the impermeable solute leads to a greater amount of intracellular counterions, thus increasing intracellular osmotic pressure.

We now turn to the question of stability. Let:

$$
\mathcal{S}=\left\{(\mathbf{c}, v) \in \mathbb{R}_{+}^{N+1} \mid \sum_{k=1}^{N} z_{k} c_{k}+z A / v=0\right\} .
$$

The dynamical system defined by (3.1) lives on this set. We must thus modify the definition of stability accordingly. A steady state of (3.1) is stable if all solutions with initial values in $\mathcal{S}$ and near the steady state stay close to the steady state. A steady state is asymptotically stable if it is stable and if all solutions with initial values in $\mathcal{S}$ and near the steady state converge to the steady state as $t \rightarrow \infty$. A steady state is globally asymptotically stable if it is stable and if all solutions starting from initial values in $\mathcal{S}$ converge to the steady state as $t \rightarrow \infty$. If we make a change of variables to obtain an $N$-dimensional dynamical system without the implicit constraint of electroneutrality, the above definitions of stability reduce to the usual ones for ODEs.

We saw in Proposition 2.1 that in the absence of active currents, the free energy $G$ defined in (2.14) is decreasing. We now construct a free energy like quantity that is decreasing in the presence of active currents. Define:

$$
\widetilde{G}=v \sum_{k=1}^{N}\left(c_{k}\left(\ln \left(\frac{c_{k}}{c_{k}^{\mathrm{e}}}\right)-1+q_{k}\right)+c_{k}^{\mathrm{e}}\right)+A\left(\ln \left(\frac{A}{v}\right)-1\right)
$$


where $q_{k}$ was defined in (3.4). For $\widetilde{G}$, we have the following analogue of Proposition 2.1.

Lemma 3.2 Let $\mathbf{c}, v$, $\phi$ satisfy system (3.1). We have:

$$
\begin{gathered}
\frac{d \widetilde{G}}{d t}=-J, \\
J=\langle\boldsymbol{\mu}+\mathbf{q}, L(\boldsymbol{\mu}+\mathbf{q})\rangle_{\mathbb{R}^{N}}+\zeta \pi_{\mathrm{w}}^{2}=\langle\boldsymbol{\gamma}+\mathbf{q}, \widehat{L}(\boldsymbol{\gamma}+\mathbf{q})\rangle_{\mathbb{R}^{N}}+\zeta \pi_{\mathrm{w}}^{2},
\end{gathered}
$$

where $\langle\cdot, \cdot\rangle_{\mathbb{R}^{N}}$ is the inner product in $\mathbb{R}^{N}$. The function $\widetilde{G}$ is thus a Lyapunov function in the sense that it is non-increasing in time.

Proof The proof is almost identical to the proof of Proposition 2.1. The second equality in the definition of $J$ comes from (3.3).

If system (3.1) has a steady state, we may rewrite (3.20) as follows. Let (c $\left.\mathbf{c}^{*}, v^{*}, \phi^{*}\right)$ be the steady state of (3.1). Note that $\pi_{\mathrm{w}}=0$ at steady state, and that $q_{k}=-\mu_{k}^{*}$ where $\mu_{k}^{*}$ is the evaluation of the chemical potential at steady state. Using this, and the fact that c satisfies the electroneutrality constraint (3.1b), we find, after some calculation:

$$
\begin{aligned}
\widehat{G}(\mathbf{c}, v) & \equiv \widetilde{G}(\mathbf{c}, v)-\widetilde{G}\left(\mathbf{c}^{*}, v^{*}\right) \\
& =v \sum_{k=1}^{N}\left(c_{k}\left(\ln \left(\frac{c_{k}}{c_{k}^{*}}\right)-1\right)+c_{k}^{*}\right)+A\left(\ln \left(\frac{v^{*}}{v}\right)-1+\frac{v}{v^{*}}\right) .
\end{aligned}
$$

The quantity $\widehat{G}$ can be interpreted as being the total free energy of the system relative to the steady state. Since $\widetilde{G}$ and $\widehat{G}$ differ only by a constant, we may replace $\widetilde{G}$ with $\widehat{G}$ in $(3.20)$ :

$$
\begin{aligned}
\frac{d \widehat{G}}{d t} & =-J=-\langle\widehat{\boldsymbol{\mu}}, L \widehat{\boldsymbol{\mu}}\rangle_{\mathbb{R}^{N}}-\zeta \pi_{\mathrm{w}}^{2}, \\
\widehat{\boldsymbol{\mu}} & =\left(\widehat{\mu}_{1}, \ldots, \widehat{\mu}_{N}\right)^{T}, \widehat{\mu}_{k}=\ln \left(\frac{c_{k}}{c_{k}^{*}}\right)+z_{k}\left(\phi-\phi^{*}\right),
\end{aligned}
$$

where we used $q_{k}=-\mu_{k}^{*}$ to rewrite $J$. Thus, if the system has a steady state, Lemma 3.2 says that the free energy relative to the steady state is always decreasing at a rate that is controlled by $\widehat{\boldsymbol{\mu}}$, the vector of chemical potential relative to the steady state. We shall find both $\widetilde{G}$ and $\widehat{G}$ useful depending on context.

In studying stability, it is sometimes convenient to use a new set of variables $\mathbf{a}=$ $\left(a_{1}, \ldots, a_{N}\right)^{T}=v \mathbf{c}, v$ and $\phi$ rather than $\mathbf{c}, v$ and $\phi$. Rewriting (3.1) in the new variables, a satisfies the differential equations:

$$
\frac{d \mathbf{a}}{d t}=-L(\boldsymbol{\mu}+\mathbf{q})
$$

We now state a result on the function $\widetilde{G}$. Note that, although the solutions $(\mathbf{c}, v)$ of (3.1) is defined only for $(\mathbf{c}, v) \in \mathbb{R}_{+}^{N+1}$, the function $\widetilde{G}(\mathbf{c}, v)$ is well-defined on 
$\overline{\mathbb{R}}_{+}^{N} \times \mathbb{R}_{+}$where $\overline{\mathbb{R}_{+}}$is the set of non-negative real numbers (overline of a set will henceforth denote its closure). The same comment applies for $\widetilde{G}$ viewed as a function of $(\mathbf{a}, v)$ and for $\widehat{G}$.

Lemma 3.3 1. Consider the function $\widetilde{G}$ defined in (3.19) and view this as a function of $(\mathbf{a}, v) \in{\overline{\mathbb{R}_{+}}}^{N} \times \mathbb{R}_{+}$where $\mathbf{a}=v \mathbf{c}$. The Hessian of $\widetilde{G}$ is positive definite at each point in $\mathbb{R}_{+}^{N+1}$ and $\widetilde{G}$ is thus a convex function.

2. Suppose condition (3.5) is satisfied. View $\widetilde{G}$ as a function of $(\mathbf{c}, v) \in \overline{\mathbb{R}}_{+}{ }^{N} \times \mathbb{R}_{+}$ and let the unique steady state of (3.1) be given by $\left(\mathbf{c}^{*}, v^{*}\right)$. Then, $\left(\mathbf{c}^{*}, v^{*}\right)$ is the unique minimizer of $\widetilde{G}$ restricted to $\overline{\mathcal{S}}$, where $\mathcal{S}$ is given in (3.18).

Proof Let $H_{G}$ denote the $(N+1) \times(N+1)$ Hessian matrix of $\widetilde{G}(\mathbf{a}, v)$. The Hessian matrix is well-defined for $(\mathbf{a}, v) \in \mathbb{R}_{+}^{N+1}$. For any vector $\mathbf{x}=\left(x_{1}, \ldots, x_{N}, x_{v}\right) \in$ $\mathbb{R}^{N+1}$ we have:

$$
\left\langle\mathbf{x}, H_{G} \mathbf{x}\right\rangle_{\mathbb{R}^{N+1}}=\sum_{k=1}^{N}\left(\frac{1}{\sqrt{a_{k}}} x_{k}-\frac{\sqrt{a_{k}}}{v} x_{v}\right)^{2}+\frac{A}{v^{2}} x_{v}^{2},
$$

The Hessian matrix $H_{G}$ is thus positive definite at every point in $(\mathbf{a}, v) \in \mathbb{R}_{+}^{N+1}$, and $\widetilde{G}$ is thus a convex function.

To prove the second item, we first rephrase the assertion in terms of $(\mathbf{a}, v)$. We must show that $\left(\mathbf{a}^{*}, v^{*}\right)=\left(v^{*} \mathbf{c}^{*}, v^{*}\right)$ is the unique minimizer of $\widetilde{G}(\mathbf{a}, v),(\mathbf{a}, v) \in$ $\overline{\mathbb{R}}_{+}^{N} \times \mathbb{R}_{+}$when restricted to the hyperplane:

$$
\sum_{k=1}^{N} z_{k} a_{k}+A=0
$$

where $\mathbf{a}=\left(a_{1}, \ldots, a_{N}\right)^{T}$.

We seek stationary points of $\widetilde{G}$ restricted to the hyperplane (3.25). Consider:

$$
\widetilde{G}_{\lambda}=\widetilde{G}+\lambda\left(\sum_{k=1}^{N} z_{k} a_{k}+A\right)
$$

where $\lambda$ is the Lagrange multiplier. The condition for a stationary point is given by:

$$
\begin{aligned}
& \frac{\partial \widetilde{G}_{\lambda}}{\partial a_{k}}=\ln \left(\frac{a_{k} / v}{c_{k}^{\mathrm{e}}}\right)+z_{k} \lambda+q_{k}=0, \\
& \frac{\partial \widetilde{G}_{\lambda}}{\partial v}=\pi_{\mathrm{w}}=0 .
\end{aligned}
$$

If we identify $\lambda$ with $\phi$, the membrane potential, the above condition is nothing other than the condition for steady state of system (3.1). Since condition (3.5) is satisfied, by Proposition 3.1, the above system has a unique solution and $(\mathbf{a}, v, \lambda)=\left(\mathbf{a}^{*}, v^{*}, \phi^{*}\right)$ 
where $\phi^{*}$ is the value of $\phi$ at the unique steady state of (3.1). Since $\widetilde{G}$ is a convex function on $\overline{\mathbb{R}}_{+} N \times \mathbb{R}_{+}$, its restriction to the hyperplane (3.25) is also a convex function. Thus, this stationary point is the unique minimizer.

We may now state our first stability result.

Proposition 3.4 Suppose condition (3.5) is satisfied. Then, the unique steady state of (3.1) is asymptotically stable. Moreover, the decay to the steady state is exponential, and the linearized operator around the steady state is diagonalizable with real and negative eigenvalues.

The linearized operator above refers to the linearization when (3.1) is seen as an ODE system on the $N$-dimensional submanifold defined by the electroneutrality constraint (3.1b). We note that asymptotic stability is in fact an immediate consequence of Lemma 3.3 by a Lyapunov stability argument. Thus, if we are only interested in asymptotic stability, there is no need to study the linearization. A Lyapunov stability argument will be used to study the global behavior of solutions in the proof of Theorem 3.5.

Proof of Proposition 3.4 It suffices to prove this claim by studying the dynamics of (3.1) in the variables $(\mathbf{a}, v)$ instead of $(\mathbf{c}, v)$. Since condition (3.5) is satisfied, there is a unique steady state by Proposition 3.1. To study the linearization around this steady state we must change variables to remove the implicit constraint (3.1b) (or equivalently, (3.25)) and obtain an $N$-dimensional ODE system.

From (3.3), we have:

$$
\frac{d \mathbf{a}}{d t}=-\widehat{L} \nabla_{a} \widetilde{G}
$$

where $\nabla_{a} \widetilde{G}$ is the gradient of $\widetilde{G}(\mathbf{a}, v)$ with respect to a while keeping $v$ fixed. Let us examine the matrix $\widehat{L}$. Take a vector $\mathbf{w} \in \mathbb{R}^{N}$. By (3.3), we have:

$$
\langle\mathbf{w}, \widehat{L} \mathbf{w}\rangle_{\mathbb{R}^{N}}=\langle\mathbf{w}, L \mathbf{w}\rangle_{\mathbb{R}^{N}}-\frac{\left(\langle\mathbf{w}, L \mathbf{z}\rangle_{\mathbb{R}^{N}}\right)^{2}}{\langle\mathbf{z}, L \mathbf{z}\rangle_{\mathbb{R}^{N}}}
$$

Since $L$ is symmetric positive definite, the above quantity is non-negative by the Cauchy-Schwarz inequality and is equal to 0 if and only if $\mathbf{w}$ is a constant multiple of $\mathbf{z}$. Thus, $\widehat{L}$ is a symmetric positive semi-definite matrix whose eigenspace corresponding to the eigenvalue 0 is spanned by $\mathbf{z}$. The restriction of $\widehat{L}$ to the orthogonal complement of this eigenspace is thus positive definite.

Consider a change of coordinates from a to an orthonormal coordinate system $\mathbf{b}$ satisfying:

$$
\mathbf{b}=\left(b_{1}, \ldots, b_{N}\right)^{T}, \quad b_{N}=\frac{1}{|\mathbf{z}|}\langle\mathbf{z}, \mathbf{a}\rangle_{\mathbb{R}^{N}} .
$$

The $N$-th coordinate axis is thus parallel to $\mathbf{z}$. Note that the electroneutrality constraint (3.25) can be written as $b_{N}=$ constant. Let $\mathbf{b}=U \mathbf{a}$, where $U$ is the orthogonal coordi- 
nate transformation matrix. In the b coordinate system, $\widehat{L}$ transforms to $\widehat{L}_{b}=U \widehat{L} U^{-1}$. Given the above properties of $\widehat{L}, \widehat{L}_{b}$ has the form:

$$
\widehat{L}_{b}=\left(\begin{array}{cc}
\widehat{L}_{b}^{\perp} & \mathbf{0}_{N-1} \\
\mathbf{0}_{N-1}^{T} & 0
\end{array}\right),
$$

where $\widehat{L}_{b}^{\perp}$ is a positive definite matrix and $\mathbf{0}_{N-1}$ is the zero column vector of length $N-1$. Rewriting (3.28) in terms of $\mathbf{b}$, we have:

$$
\frac{d \mathbf{b}}{d t}=-\widehat{L}_{b} \nabla_{b} \widetilde{G}
$$

where $\nabla_{b} \widetilde{G}$ is the gradient of $\widetilde{G}$ (keeping $v$ fixed) seen as a function of $\mathbf{b}$. From (3.31), we see that $b_{N}$ remains constant. Let $\widehat{\mathbf{b}}=\left(b_{1}, \ldots, b_{N-1}\right)^{T}$. We have:

$$
\frac{d \widehat{\mathbf{b}}}{d t}=-\widehat{L}_{b}^{\perp} \nabla_{\widehat{b}} \widetilde{G}
$$

where $\nabla_{\widehat{b}}$ is the gradient of $\widetilde{G}$ with respect to $\widehat{\mathbf{b}}$ while keeping $b_{N}$ and $v$ fixed. We have thus reduced the system (3.1) to (3.33) and to (3.1c) which can be written as:

$$
\frac{d v}{d t}=-\zeta \frac{\partial \widetilde{G}}{\partial v}
$$

Letting $\mathbf{u}=\left(b_{1}, \ldots, b_{N-1}, v\right)^{T}$, we may write our system as follows:

$$
\frac{d \mathbf{u}}{d t}=-L_{u} \nabla_{u} \widetilde{G}, \quad L_{u}=\left(\begin{array}{cc}
\widehat{L}_{b}^{\perp} & \mathbf{0}_{N-1} \\
\mathbf{0}_{N-1}^{T} & \zeta
\end{array}\right),
$$

where $\nabla_{u}$ is the gradient of $\widetilde{G}$ with respect to $\mathbf{u}$ while keeping $b_{N}$ fixed. We have thus obtained the requisite $N$-dimensional ODE system in the variables $\mathbf{u}$; the electroneutrality constraint $b_{N}=$ constant only appears as a parameter of the system.

Let $H_{u}$ be the Hessian of $\widetilde{G}$ with respect to $\mathbf{u}$. Given Lemma $3.3, H_{u}$ is symmetric positive definite. Indeed, the quadratic form defined by $H_{u}$ is just the restriction of $H_{G}$ (defined in Lemma 3.3) to the subspace of $\mathbb{R}^{N+1}$ orthogonal to $\left(\mathbf{z}^{T}, 0\right)^{T}$. The linearized operator of (3.35) around steady state is thus given by $-L_{u} H_{u}^{*}$ where $H_{u}^{*}$ is the evaluation of $H_{u}$ at the steady state. Note that $L_{u} H_{u}^{*}$ is similar to $\left(H_{u}^{*}\right)^{1 / 2} L_{u}\left(H_{u}^{*}\right)^{1 / 2}$ where $\left(H_{u}^{*}\right)^{1 / 2}$ is the positive square root of $H_{u}^{*}$, which exists thanks to positive definiteness of $H_{u}^{*}$. Since $\left(H_{u}^{*}\right)^{1 / 2} L_{u}\left(H_{u}^{*}\right)^{1 / 2}$ is a symmetric matrix and since $L_{u}$ is symmetric positive definite, so is $\left(H_{u}^{*}\right)^{1 / 2} L_{u}\left(H_{u}^{*}\right)^{1 / 2}$. Thus, $-L_{u} H_{u}^{*}$ is diagonalizable with real negative eigenvalues. The steady state is asymptotically stable and the approach to steady state is exponential.

By rewriting (3.1) as (3.35), we see that the system is a gradient flow on the hyperplane defined by the electroneutrality constraint where the metric is given by $L_{u}^{-1}$. This led us to the conclusion that the linearization is diagonalizable with real negative 
eigenvalues. Note that we made essential use of the symmetry of the matrix $L$, which came from the Onsager reciprocity principle (see (2.27)). The gradient structure of our system combined with the convexity of $\widetilde{G}$ has another interesting consequence as we shall see in Lemma 3.6.

\subsection{Global behavior}

We now state the main result of this Section.

Theorem 3.5 Suppose condition (3.5) is satisfied. Then, (3.1) has a unique steady state and it is globally asymptotically stable. The linearization around steady state is diagonalizable with real negative eigenvalues.

Existence of the unique steady state was proved in Proposition 3.1. Asymptotic stability and the property of the linearized operator was proved in Proposition 3.4. We have thus only to prove that all solutions with initial value in $\mathcal{S}$ (see (3.18)) converge to the steady state as $t \rightarrow \infty$. Implicit in this assertion is that these solutions are global (defined for all positive time). Once this is established, we use the fact that $\widehat{G}$ is a Lyapunov function to obtain the desired result.

To prove that all solutions are global, we must rule out two possibilities. The first is that the solution may grow unbounded in finite time. To show that this is not possible, we make use of the function $\widehat{G}$. The second possibility is that one or more of the concentrations $c_{k}$ or the cell volume $v$ may come arbitrarily close to 0 in finite time. To show that this cannot happen, we examine the free energy dissipation function $J$ defined in (3.20).

Lemma 3.6 View $J$ defined in (3.20) as a function of $(\mathbf{c}, v) \in \mathbb{R}_{+}^{N}$ :

$$
J(\mathbf{c}, v)=J_{c}(\mathbf{c})+\zeta\left(\pi_{\mathrm{w}}(\mathbf{c}, v)\right)^{2}, J_{c}(\mathbf{c})=\langle\boldsymbol{\gamma}+\mathbf{q}, \widehat{L}(\boldsymbol{\gamma}+\mathbf{q})\rangle_{\mathbb{R}^{N}}
$$

1. Consider any solution $(\mathbf{c}(t), v(t))$ of system (3.1). We have:

$$
\begin{aligned}
\frac{d}{d t} J(\mathbf{c}(t), v(t)) & =-\frac{2}{v} \sum_{k=1}^{N}\left(\frac{\rho_{k}}{\sqrt{c_{k}}}-\zeta \pi_{\mathrm{w}} \sqrt{c_{k}}\right)^{2}-\frac{2 A}{v^{2}}\left(\zeta \pi_{\mathrm{W}}\right)^{2}, \\
\rho & =\left(\rho_{1}, \ldots, \rho_{N}\right)^{T}=\widehat{L}(\boldsymbol{\gamma}+\mathbf{q}) .
\end{aligned}
$$

The function $J$ is thus a Lyapunov function in the sense that it is monotone nonincreasing.

2. The function $J_{c}(\mathbf{c})$ defined in (3.36) tends to $+\infty$ as $\mathbf{c}$ approaches any point on $\partial \mathbb{R}_{+}^{N}$ where $\partial \cdot$ denotes the boundary of a set.

That $J$ is a Lyapunov function can be seen as follows. View $J$ as a function of $\mathbf{u}$ introduced in the proof of Proposition 3.4. First, note that:

$$
\frac{d \widetilde{G}}{d t}=-\left\langle\nabla_{u} \widetilde{G}, \frac{d \mathbf{u}}{d t}\right\rangle_{\mathbb{R}^{N}}=-\left\langle\nabla_{u} \widetilde{G}, L_{u} \nabla_{u} \widetilde{G}\right\rangle_{\mathbb{R}^{N}}=-J
$$


where we used (3.35) in the second equality. We thus have:

$$
\begin{aligned}
\frac{d J}{d t} & =2\left\langle L_{u} \nabla_{u} \widetilde{G}, \frac{d}{d t}\left(\nabla_{u} \widetilde{G}\right)\right\rangle_{\mathbb{R}^{N}}=2\left\langle L_{u} \nabla_{u} \widetilde{G}, H_{u} \frac{d \mathbf{u}}{d t}\right\rangle_{\mathbb{R}^{N}} \\
& =-2\left\langle L_{u} \nabla_{u} \widetilde{G}, H_{u} L_{u} \nabla_{u} \widetilde{G}\right\rangle_{\mathbb{R}^{N}}
\end{aligned}
$$

where $H_{u}$ is the Hessian matrix of $\widetilde{G}$ with respect to $\mathbf{u}$. We used (3.35) in the last equality. As we saw in the proof of Proposition 3.4, $H_{u}$ is positive definite. Therefore, $J$ is monotone non-increasing. We see that the Lyapunov property of $J$ is a general consequence of the fact our system, in suitable variables, is a gradient flow of the convex energy function $\widetilde{G}$.

Proof of Lemma 3.6 We saw above that $d J / d t$ is non-positive, but we have not obtained the right hand expression in (3.37). This is most easily done by direct calculation. We turn to the second claim. Take any point $\mathbf{c}^{\mathrm{b}} \in \partial \mathbb{R}_{+}^{N}$ and assume without loss of generality that the first $1 \leq l \leq N$ components of $\mathbf{c}^{\mathrm{b}}$ are 0 :

$$
\mathbf{c}^{\mathrm{b}}=(\underbrace{0, \ldots, 0}_{l}, c_{l+1}^{\mathrm{b}}, \ldots, c_{N}^{\mathrm{b}}) .
$$

Decompose the vector $\boldsymbol{\gamma}+\mathbf{q}$ in the following fashion.

$$
\begin{aligned}
\boldsymbol{\gamma}+\mathbf{q} & =-\left(\boldsymbol{\gamma}_{1}+\boldsymbol{\gamma}_{2}\right), \\
\boldsymbol{\gamma}_{1} & =-(\gamma_{1}+q_{1}, \ldots, \gamma_{l}+q_{l}, \underbrace{0, \ldots, 0}_{N-l})^{T} \\
\boldsymbol{\gamma}_{2} & =-(\underbrace{0, \ldots, 0}_{l}, \gamma_{l+1}+q_{l+1}, \ldots, \gamma_{N}+q_{N})^{T} .
\end{aligned}
$$

Now, consider a sequence of points $\mathbf{c}^{n} \in \mathbb{R}_{+}^{N}, n=1,2, \ldots$ such that $\mathbf{c}^{n} \rightarrow \mathbf{c}^{\mathrm{b}}$ as $n \rightarrow \infty$. Given that:

$$
\gamma_{k}+q_{k}=\ln \left(\frac{c_{k}}{c_{k}^{\mathrm{e}}}\right)+q_{k}, \quad k=1, \ldots, N,
$$

each of the first $l$ non-zero components of $\boldsymbol{\gamma}_{1}$ goes to $+\infty$ as $\mathbf{c}^{n} \rightarrow \mathbf{c}^{\mathrm{b}}$ whereas $\boldsymbol{\gamma}_{2}$ remains bounded as $\mathbf{c}^{n} \rightarrow \mathbf{c}^{\mathrm{b}}$. Now, take an arbitrary vector

$$
\mathbf{w}=\left(w_{1}, \ldots, w_{N}\right) \in \mathbb{R}^{N},|\mathbf{w}|=1, w_{k} \geq 0, \quad k=1, \ldots, N
$$

Recall from (3.29) and the subsequent discussion that $\langle\mathbf{w}, \widehat{L} \mathbf{w}\rangle_{\mathbb{R}^{N}}$ is positive if $\mathbf{w}$ is not parallel to $\mathbf{z}$. The vectors $\mathbf{w}$ and $\mathbf{z}$ are indeed not parallel since $\mathbf{z}$ must have at least one component that is negative (there is at least one ionic species with negative valence). Therefore,

$$
\min _{w_{k} \geq 0, k=1, \ldots, N,|\mathbf{w}|=1}\langle\mathbf{w}, \widehat{L} \mathbf{w}\rangle_{\mathbb{R}^{N}} \equiv K_{w}>0
$$


given that the set satisfying $w_{k} \geq 0, k=1, \ldots, N,|\mathbf{w}|=1$ is compact. Therefore, for any vector $\mathbf{u}$ whose components are non-negative, we have:

$$
\langle\mathbf{u}, \widehat{L} \mathbf{u}\rangle_{\mathbb{R}^{N}} \geq K_{w}|\mathbf{u}|^{2}
$$

Now, let us take the limit of $J_{c}(\mathbf{c})$ as $\mathbf{c}^{n} \rightarrow \mathbf{c}^{\mathrm{b}}$. If $\mathbf{c}^{n}$ is sufficiently close to $\mathbf{c}^{\mathrm{b}}$, the first $l$ components of $\boldsymbol{\gamma}_{1}$ as defined in (3.41) are positive. Therefore, we have:

$$
\begin{aligned}
J_{c}\left(\mathbf{c}^{n}\right) & \geq\left\langle\boldsymbol{\gamma}_{1}+\boldsymbol{\gamma}_{2}, \widehat{L}\left(\boldsymbol{\gamma}_{1}+\boldsymbol{\gamma}_{2}\right)\right\rangle_{\mathbb{R}^{N}} \\
& \geq\left(K_{w}\left|\boldsymbol{\gamma}_{1}\right|-2\left|\widehat{L} \boldsymbol{\gamma}_{2}\right|\right)\left|\boldsymbol{\gamma}_{1}\right|+\left\langle\boldsymbol{\gamma}_{2}, \widehat{L} \boldsymbol{\gamma}_{2}\right\rangle_{\mathbb{R}^{N}}
\end{aligned}
$$

where we used (3.45) and the Cauchy-Schwarz inequality. Since $\left|\boldsymbol{\gamma}_{1}\right| \rightarrow+\infty$ and $\boldsymbol{\gamma}_{2}$ remains bounded as $\mathbf{c}^{n} \rightarrow \mathbf{c}^{\mathrm{b}}, J_{c}\left(\mathbf{c}^{n}\right)$ tends to $+\infty$.

Proof of Theorem 3.5 Take an arbitrary initial value $\left(\mathbf{c}^{0}, v^{0}\right) \in \mathcal{S}$ where $\mathcal{S}$ was defined in (3.18). We first show that the solution to (3.1) starting from $\left(\mathbf{c}^{0}, v^{0}\right)$ is defined for all $t>0$.

View $\widehat{G}$ of (3.21) as a function of $(\mathbf{c}, v)$. Consider the set:

$$
\mathcal{A}_{M}=\{(\mathbf{c}, v) \in \mathcal{S} \mid \widehat{G}(\mathbf{c}, v)<M\}
$$

where we choose $M$ so that $M>\widehat{G}\left(\mathbf{c}^{0}, v^{0}\right)$. Given (3.22), the solution stays within $\mathcal{A}_{M}$ so long as the solution is defined. We first show that the set $\mathcal{A}_{M}$ is bounded and that it is bounded away from the hyperplane $v=0$.

Any element in $\mathcal{A}_{M}$ satisfies:

$$
\sum_{k=1}^{N}\left(c_{k}\left(\ln \left(\frac{c_{k}}{c_{k}^{*}}\right)-1\right)+c_{k}^{*}\right)<-\frac{A}{v}\left(\ln \left(\frac{v^{*}}{v}\right)-1\right)-\frac{A}{v^{*}}+\frac{M}{v} .
$$

It is easily seen that the left hand side of the above is greater than or equal to 0 . Therefore the right hand side must be greater than 0 , from which we obtain:

$$
A\left(\ln \left(\frac{v^{*}}{v}\right)-1+\frac{v}{v^{*}}\right)<M
$$

Therefore, $v$ must satisfy $0<v_{-}<v<v_{+}<\infty$ for some constants $v_{+}$and $v_{-}$. Let $\bar{M}$ be the supremum of the right hand side of (3.48) over $v_{-}<v<v_{+}$. This $\bar{M}$ is clearly finite. Thus,

$$
\sum_{k=1}^{N}\left(c_{k}\left(\ln \left(\frac{c_{k}}{c_{k}^{*}}\right)-1\right)+c_{k}^{*}\right) \leq \bar{M}
$$


We thus see that $c_{k}$ must be bounded above by a constant $c_{+}$that depends only on $M$. Therefore, we have:

$$
0<v_{-}<v<v_{+}, \quad c_{k}<c_{+}, \quad k=1, \ldots, N
$$

Let $(\mathbf{c}(t), v(t))$ be the solution to (3.1) with initial data $\left(\mathbf{c}^{0}, v^{0}\right)$. Since the solution stays within $\mathcal{A}_{M}$, we know that the solution satisfies the bound (3.51). We now show that the concentrations $c_{k}(t)$ are bounded away from 0. Recall from (3.37) of Lemma 3.6 that the function $J$ is a non-increasing function in time. Thus, $J(\mathbf{c}(t), v(t))$ $\leq J\left(\mathbf{c}^{0}, v^{0}\right)=M_{J}$. Since $J_{c}(\mathbf{c}) \leq J(\mathbf{c}, v)$, we have $J_{c}(\mathbf{c}(t)) \leq M_{J}$. By the second item in Lemma 3.6, the set:

$$
\left\{\mathbf{c}=\left(c_{1}, \ldots, c_{N}\right) \in \mathbb{R}_{+}^{N} \mid J_{c}(\mathbf{c}) \leq M_{J}, c_{k}<c_{+}, \quad k=1, \ldots N\right\}
$$

must be bounded away from $\partial \mathbb{R}_{+}^{N}$. Therefore, we have:

$$
0<c_{-}<c_{k}(t)<c_{+}, \quad k=1, \ldots, N
$$

where $c_{-}$is a constant that depends only on $M$ and $M_{J}$. This, together with (3.51), implies that the solution $(\mathbf{c}(t), v(t))$ lies in a compact subset $\mathcal{K}$ of $\mathcal{S}$. This shows that the solution must be defined for all time.

We now show that the solution $(\mathbf{c}(t), v(t))$ converges to the steady state $\left(\mathbf{c}^{*}, v^{*}\right)$. Take an arbitrary $\epsilon>0$ and let $\mathcal{B}_{\epsilon} \subset \mathbb{R}^{N+1}$ be the open ball of radius $\epsilon$ centered at $\left(\mathbf{c}^{*}, v^{*}\right)$. We must show that $(\mathbf{c}(t), v(t)) \in \mathcal{B}_{\epsilon}$ after finite time. Observe that we can make $\delta>0$ sufficiently small so that $\mathcal{A}_{\delta} \subset \mathcal{B}_{\epsilon}\left(\mathcal{A}_{\delta}\right.$ is defined by replacing $M$ with $\delta$ in (3.47)). This is clear since, by Lemma $3.3,\left(\mathbf{c}^{*}, v^{*}\right)$ is the unique minimizer of $\widehat{G}$ over $\overline{\mathcal{S}}$.

Take $\delta$ so small that $\mathcal{A}_{\delta} \in \mathcal{B}_{\epsilon}$. If $M \leq \delta, \mathcal{A}_{M} \subset \mathcal{A}_{\delta} \subset \mathcal{B}_{\epsilon}$. Since the solution is contained in $\mathcal{A}_{M}$, there is nothing to prove. Assume $M>\delta$. We would like to show that the solution is contained in $\mathcal{A}_{\delta} \subset \mathcal{B}_{\epsilon}$ after finite time. We prove this by contradiction. Suppose the solution never enters $\mathcal{A}_{\delta}$. Recall that the solution $(\mathbf{c}(t), v(t))$ was contained in a compact set $\mathcal{K} \subset \mathcal{S}$. The function $J(\mathbf{c}, v)$ is clearly positive on $\mathcal{K} \backslash \mathcal{A}_{\delta}$, since $\left(\mathbf{c}^{*}, v^{*}\right) \in \mathcal{A}_{\delta}$ is the only point at which $J=0$. Since $\mathcal{K} \backslash \mathcal{A}_{\delta}$ is a compact set, $J>K_{J}>0$ on $\mathcal{K} \backslash \mathcal{A}_{\delta}$ where $K_{J}$ is a positive constant. By (3.20) of Lemma 3.2 (or equivalently, (3.22)), we see that:

$$
\widehat{G}(\mathbf{c}(t), v(t))<M-K_{J} t .
$$

This implies that the solution will be in the set $\mathcal{A}_{\delta}$ for $t>(M-\delta) / K_{J}$, a contradiction.

Theorem 3.5 shows that system (3.1) has the following remarkable robustness property. Suppose the pump rates $\mathbf{p}$ and the extracellular concentrations $\mathbf{c}^{\mathrm{e}}$ are perturbed within the bounds of condition (3.5). Then, the cell will approach the new global steady state. 
The next theorem shows that when the condition (3.5) is not met, the cell volume $v(t) \rightarrow \infty$ as $t \rightarrow \infty$. There is thus a dichotomy in the behavior of system (3.1) depending on whether the condition (3.5) is satisfied.

Theorem 3.7 Suppose condition (3.5) is not satisfied so that $f_{\min }\left(\mathbf{q}, \mathbf{c}^{\mathrm{e}}, \mathbf{z}\right) \geq 0$. Take any solution $(\mathbf{c}(t), v(t))$ to $(3.1)$.

1. Suppose $f_{\min }\left(\mathbf{q}, \mathbf{c}^{\mathrm{e}}, \mathbf{z}\right)>0$. Then,

$$
\lim _{t \rightarrow \infty} v(t)=\infty
$$

2. Suppose $f_{\min }\left(\mathbf{q}, \mathbf{c}^{\mathrm{e}}, \mathbf{z}\right)=0$. Let $\phi_{\min }$ be as in Proposition 3.1. Then,

$$
\begin{aligned}
\lim _{t \rightarrow \infty} c_{k}(t) & =c_{k}^{\mathrm{e}} \exp \left(-q_{k}-z_{k} \phi_{\min }\right), \quad k=1, \ldots N \\
\lim _{t \rightarrow \infty} v(t) & =\infty
\end{aligned}
$$

Proof We shall work with the variables $\mathbf{c}$ and $w=1 / v$. In these variables, (3.1) can be written as:

$$
\begin{aligned}
\frac{d \mathbf{c}}{d t} & =w\left(-\widehat{L}(\boldsymbol{\gamma}+\mathbf{q})+\zeta \pi_{\mathrm{w}} \mathbf{c}\right), \\
0 & =\sum_{k=1}^{N} z_{k} c_{k}+z A w=\sum_{k=1}^{N} z_{k} c_{k}^{\mathrm{e}}, \\
\frac{d w}{d t} & =w^{2} \zeta \pi_{\mathrm{w}} .
\end{aligned}
$$

The solutions are defined on the set:

$$
\mathcal{T}=\left\{(\mathbf{c}, w) \in \mathbb{R}_{+}^{N+1} \mid \sum_{k=1}^{N} z_{k} c_{k}+z A w=0\right\}
$$

Note that $\mathcal{T}$ is just the set $\mathcal{S}$ of (3.18) written in the (c, $w$ ) coordinates. Take any initial data $\left(\mathbf{c}^{0}, w^{0}\right) \in \mathcal{T}$ and let $(\mathbf{c}(t), w(t))$ be the solution to (3.57) starting from this point. Showing that $v(t) \rightarrow \infty$ is equivalent to showing that $w(t) \rightarrow 0$. We divide the proof into several steps.

Step 1 View $\widetilde{G}$ defined in (3.20) as a function of (c, $w)$. Consider the set:

$$
\mathcal{A}_{M}=\left\{(\mathbf{c}, w) \in \mathbb{R}_{+}^{N+1} \mid \sum_{k=1}^{N} z_{k} c_{k}+z A w=0, \widetilde{G}(\mathbf{c}, w)<M\right\} .
$$

This is the same set as (3.47) except that we use the function $\widetilde{G}(\mathbf{c}, w)$ instead of $\widehat{G}(\mathbf{c}, v)$. We prove that $\mathcal{A}_{M}$ is a bounded set. 
For any point in $\mathcal{A}_{M}$, we have:

$$
\sum_{k=1}^{N}\left(c_{k}\left(\ln \left(\frac{c_{k}}{c_{k}^{\mathrm{e}}}\right)-1+q_{k}\right)+c_{k}^{\mathrm{e}}\right)<M w-A w(\ln (A w)-1) .
$$

Since the left hand side is bounded from below, there is some positive constant $m$, independent of $M$, such that:

$$
-m<M w-A w(\ln (A w)-1) .
$$

Let:

$$
g(w)=-\frac{m}{w}+A(\ln (A w)-1)
$$

The function $g(w)$ is a monotone increasing function in $w$ such that $g(w) \rightarrow-\infty$ as $w \rightarrow 0$ and $g(w) \rightarrow \infty$ as $w \rightarrow \infty$. Let $w_{+}(M)=g^{-1}(M)$. Given (3.61), we have:

$$
0<w<w_{+}(M) \text { for any }(\mathbf{c}, w) \in \mathcal{A}_{M} \text {. }
$$

Since $w$ is bounded between 0 and $w_{+}(M)$, we see from (3.60) that $c_{k}$ must also be bounded in $\mathcal{A}_{M}$ :

$$
0<c_{k}<c_{+}(M), \quad k=1, \ldots, N \quad \text { for any }(\mathbf{c}, w) \in \mathcal{A}_{M} .
$$

Step 2 We prove that the solution $(\mathbf{c}(t), w(t))$ is defined for all positive time. Choose $M=M_{0}$ so that $M_{0}>\widetilde{G}\left(\mathbf{c}_{0}, w_{0}\right)$. Suppose that the solution exists only up to $t<T_{0}, T_{0}<\infty$. Since $\widetilde{G}$ is monotone non-increasing, we have $(\mathbf{c}(t), w(t)) \in$ $\mathcal{A}_{M_{0}}, t<T_{0}$. Since $\mathcal{A}_{M_{0}}$ is bounded by (3.63) and (3.64), there is a sequence of times $t_{1}<t_{2} \cdots \rightarrow T_{0}$ such that $\mathbf{x}\left(t_{n}\right)=\left(\mathbf{c}\left(t_{n}\right), w\left(t_{n}\right)\right) \rightarrow \mathbf{x}^{\mathrm{b}}=\left(\mathbf{c}^{\mathrm{b}}, w^{\mathrm{b}}\right) \in \partial \mathcal{A}_{M_{0}}$ as $n \rightarrow \infty$. The limit point $\mathbf{x}^{\mathrm{b}}$ cannot be in $\mathbb{R}_{+}^{N+1}$ since, if so, the solution can be continued beyond time $T_{0}$. We also see that $c_{k}, k=1, \ldots, N$ must stay away from 0 by an argument using Lemma 3.6 similarly to the proof of Theorem 3.5. This implies that $\mathbf{c}^{\mathrm{b}} \in \mathbb{R}_{+}^{N}$ and $w^{\mathrm{b}}=0$. By (3.1c), we have:

$$
\frac{d}{d t}\left(\frac{1}{w}\right)=\zeta\left(\sum_{k=1}^{N}\left(c_{k}-c_{k}^{\mathrm{e}}\right)+A w\right)
$$

Since the right hand side of the above is bounded in $\mathcal{A}_{M_{0}}$ by (3.63) and (3.64), $1 / w(t)$ remains finite in finite time. Thus, $w\left(t_{n}\right) \rightarrow 0$ is impossible as $t_{n} \rightarrow T_{0}<\infty$. We have a contradiction.

Step 3 Let $\mathcal{O}$ be the orbit:

$$
\mathcal{O}=\{(\mathbf{c}(t), w(t)) \in \mathcal{T}, t \geq 0\}
$$


We would like to see whether

$$
\underline{J}=\inf _{(\mathbf{c}, w) \in \mathcal{O}} J(\mathbf{c}, w)
$$

is positive. Recall that $J=0$ in $\mathcal{T}$ if and only if the point $(\mathbf{c}, w)$ is a steady state of (3.1). Given our assumption that (3.5) is not satisfied, by Proposition 3.1, a steady state does not exist. Therefore, $J>0$ in $\mathcal{O} \subset \mathcal{T}$. Thus, if $\underline{J}=0$, since $\mathcal{O}$ is a bounded set, there is a sequence of points $\mathbf{x}^{n} \in \mathcal{O}, n=1,2, \ldots$ that approaches a point $\mathbf{x}^{\infty}=\left(\mathbf{c}^{\infty}, w^{\infty}\right) \in \overline{\mathcal{T}}$ such that $J\left(\mathbf{x}^{n}\right) \rightarrow 0$ as $n \rightarrow \infty$. The limit point $\mathbf{x}^{\infty}$ cannot be in $\mathcal{T} \in \mathbb{R}_{+}^{N+1}$ since $J>0$ there. Since $\mathbf{c}(t)$ stays away from $\partial \mathbb{R}_{+}^{N}, \mathbf{c}^{\infty} \notin \partial \mathbb{R}_{+}^{N}$. Thus, $\mathbf{c}^{\infty} \in \mathbb{R}_{+}^{N}$ and $w^{\infty}=0$. Since the function $J$ is continuous up to points $(\mathbf{c}, w)=\left(\mathbf{c}^{\infty}, 0\right), \mathbf{c}^{\infty} \in \mathbb{R}_{+}^{N}$, we examine the positivity of $J$ on the set:

$$
\mathcal{R}=\left\{\mathbf{x}=(\mathbf{c}, w) \in \partial \mathcal{T} \mid \mathbf{c} \in \mathbb{R}_{+}^{N}, w=0\right\}
$$

On $\mathcal{R}, J$ can be written as:

$$
J(\mathbf{c}, w=0)=J_{c}(\mathbf{c})+\zeta\left(\pi_{\mathrm{w}}^{0}(\mathbf{c})\right)^{2}, \pi_{\mathrm{w}}^{0}(\mathbf{c})=\sum_{k=1}^{N}\left(c_{k}^{\mathrm{e}}-c_{k}\right)
$$

We see that $J=0$ if and only if $J_{c}(\mathbf{c})=0$ and $\pi_{\mathrm{w}}^{0}=0$. It is easily seen that $J_{c}(\mathbf{c})=0$ in $\mathcal{R}$ if and only if $\mathbf{c}=\mathbf{c}^{*}$ where $\mathbf{c}^{*}$ is given by:

$$
\mathbf{c}^{*}=\left(c_{1}^{*}, \ldots, c_{N}^{*}\right)^{T}, c_{k}^{*} \equiv c_{k}^{\mathrm{e}} \exp \left(-q_{k}-z_{k} \phi_{\min }\right), \quad k=1, \ldots N
$$

where $\phi_{\min }$ is as defined in the statement of Proposition 3.1. Let us evaluate $\pi_{\mathrm{w}}^{0}$ at this point. Substituting (3.70) into (3.69) and recalling the definition of $f_{\min }$ in (3.5),

$$
\pi_{\mathrm{w}}^{0}\left(\mathbf{c}^{*}\right)=-f_{\min }\left(\mathbf{q}, \mathbf{c}^{\mathrm{e}}, \mathbf{z}\right)
$$

Therefore, we have:

$$
\underline{J}>0 \text { if } f_{\min }>0 .
$$

When $f_{\min }=0$, we have the following. Let $\mathcal{B}_{\eta}$ be the open ball of radius $\eta>0$ centered at $\left(\mathbf{c}^{*}, 0\right)$. Then,

$$
\underline{J}_{\eta} \equiv \inf _{(\mathbf{c}, w) \in \mathcal{O} \backslash \mathcal{B}_{\eta}} J(\mathbf{c}, w)>0
$$

Step 4 We prove our claim when $f_{\min }>0$. Given that $(\mathbf{c}(t), w(t)) \in \mathcal{O}$, by Lemma 3.2 we have:

$$
\widetilde{G}(\mathbf{c}(t), w(t))<M_{0}-\underline{J} t .
$$


By (3.63), we have:

$$
0<w(t)<w^{+}\left(M_{0}-\underline{J} t\right)
$$

Note that $\underline{J}>0$ by (3.72). Since $w^{+}(M) \rightarrow 0$ as $M \rightarrow-\infty$, we see from (3.75) that $w(t) \rightarrow 0$ as $t \rightarrow \infty$. This proves (3.55).

Step 5 In the rest of the proof, we study the $f_{\min }=0$ case. As an initial step, we prove the following. For any $\eta>0$, there is a time $t_{\eta} \geq 0$ such that the point $\left(\mathbf{c}\left(t_{\eta}\right), w\left(t_{\eta}\right)\right)$ is in $\mathcal{B}_{\eta}$. We prove this by contradiction. Suppose otherwise. Then, there is an $\eta>0$ such that $\mathcal{B}_{\eta} \cap \mathcal{O}$ is empty.

We first show that $\widetilde{G}$ is bounded from below in $\mathcal{O}$ by a constant $M_{\eta}$. Suppose otherwise. Then, there is a sequence of points $\mathbf{x}^{n}=\left(\mathbf{c}^{n}, w^{n}\right) \in \mathcal{O}, n=1,2, \ldots$ converging to $\mathbf{x}^{\infty}=\left(\mathbf{c}^{\infty}, w^{\infty}\right) \in \overline{\mathcal{O}}$ such that $\widetilde{G}\left(\mathbf{c}^{n}, w^{n}\right) \rightarrow-\infty$. Since $\widetilde{G}(\mathbf{c}, w)$ is a continuous function for $\mathbf{c} \in \overline{\mathbb{R}}_{+} N, w>0$, the only possibility is that $w^{\infty}=0$. Write $\widetilde{G}$ as:

$$
\begin{aligned}
\widetilde{G}(\mathbf{c}, w) & =\frac{1}{w} \rho(\mathbf{c})+A(\ln (A w)-1), \\
\rho(\mathbf{c}) & =\sum_{k=1}^{N}\left(c_{k}\left(\ln \left(\frac{c_{k}}{c_{k}^{\mathrm{e}}}\right)-1+q_{k}\right)+c_{k}^{\mathrm{e}}\right) .
\end{aligned}
$$

It suffices to show that $\rho\left(\mathbf{c}^{\infty}\right)>0$. If this is true, we see from (3.76) that $\widetilde{G}\left(\mathbf{c}^{n}, w^{n}\right) \rightarrow$ $\infty$, contradicting our assumption that $\widetilde{G}\left(\mathbf{c}^{n}, w^{n}\right) \rightarrow-\infty$. It is easily seen by a calculation identical to the proof of Lemma 3.3 that the unique minimizer of $\rho(\mathbf{c})$ under the constraint

$$
\sum_{k=1}^{N} z_{k} c_{k}=0 .
$$

is $\mathbf{c}=\mathbf{c}^{*}$, at which point $\rho\left(\mathbf{c}^{*}\right)=0$. Given that $\left(\mathbf{c}^{\infty}, 0\right) \notin \mathcal{B}_{\eta}$, we see that $\mathbf{c}^{\infty} \neq \mathbf{c}^{*}$, and thus $\rho\left(\mathbf{c}^{\infty}\right)>0$.

By Lemma 3.2 and using (3.73), we have:

$$
\widetilde{G}(\mathbf{c}(t), w(t)) \leq M_{0}-\underline{J}_{\eta} t
$$

so long as $(\mathbf{c}(t), w(t)) \notin \mathcal{B}_{\eta}$. Note that $\underline{J}_{\eta}>0$ by (3.73). Thus, if $t>\left(M_{0}-M_{\eta}\right) / \underline{J}_{\eta}$, then $\widetilde{G}<M_{\eta}$, which contradicts our result that $\widetilde{G}$ must be greater than $M_{\eta}$ on $\mathcal{O}$.

Step 6 We would like to show that there is a positive number $\eta>0$ such that any solution with initial data in $\mathcal{B}_{\eta} \cap \mathcal{T}$ will converge to $\left(\mathbf{c}^{*}, 0\right)$ as $t \rightarrow \infty$. If this is true, we can combine this with the result of Step 5 to immediately conclude that that solutions of (3.57) converge to $(\mathbf{c}, w)=\left(\mathbf{c}^{*}, 0\right)$ as $t \rightarrow \infty$. This would prove (3.56).

The vector field defined by the right hand sides of (3.57a) and (3.57c) is degenerate at $w=0$. Rescaling the vector field by a positive scalar factor does not alter the solution orbits, so we shall study the behavior of an appropriately rescaled system 
(Chicone 1999; Benson et al. 2010). Rescale (3.57a) and (3.57c) by a factor of $1 / w$. This removes the degeneracy at $w=0$ :

$$
\begin{aligned}
\frac{d \mathbf{c}}{d \tau} & =-\widehat{L}(\boldsymbol{\gamma}+\mathbf{q})+\zeta \pi_{\mathrm{w}} \mathbf{c}, \\
\frac{d w}{d \tau} & =w \zeta \pi_{\mathrm{w}} .
\end{aligned}
$$

We have taken the time parameter to be $\tau$ to distinguish the solutions of this system with those of (3.57). We consider (3.79) on the set:

$$
\mathcal{T}^{\prime}=\left\{(\mathbf{c}, w) \in \mathbb{R}_{+}^{N} \times \overline{\mathbb{R}_{+}} \mid \sum_{k=1}^{N} z_{k} c_{k}+z A w=0\right\}
$$

The difference between $\mathcal{T}$ and $\mathcal{T}^{\prime}$ is whether or not $w$ is allowed to be equal to 0 .

If we can show that any solution to (3.79) with initial data in $\mathcal{B}_{\eta} \cap \mathcal{T}$ converges to $\left(\mathbf{c}^{*}, 0\right)$ as $\tau \rightarrow \infty$, the same is true for (3.57) as $t \rightarrow \infty$. This can be seen as follows. Let $(\mathbf{c}(t), w(t))$ be the solution to (3.57) with initial data $\left(\mathbf{c}^{0}, w^{0}\right) \in \mathcal{B}_{\eta} \cap \mathcal{T}$ and $(\widetilde{\mathbf{c}}(\tau), \widetilde{w}(\tau))$ be the solution to (3.79) with the same initial conditions. Define the function $\xi(t)$ with:

$$
t=\int_{0}^{\xi(t)} \frac{1}{\widetilde{w}(\tau)} d \tau
$$

This function is well-defined since $\widetilde{w}(\tau)>0$. This positivity is a simple consequence of the backward uniqueness of solutions. The expressions $\widetilde{\mathbf{c}}(\xi(t))$ and $\widetilde{w}(\xi(t))$ satisfy (3.57), and thus, by uniqueness of solutions:

$$
(\mathbf{c}(t), w(t))=(\widetilde{\mathbf{c}}(\xi(t)), \widetilde{w}(\xi(t)))
$$

By (3.81) and the fact that $\widetilde{w}(\tau) \rightarrow 0$ as $\tau \rightarrow \infty$, we see that $\xi \rightarrow \infty$ whenever $t \rightarrow \infty$. Therefore,

$$
\lim _{t \rightarrow \infty}(\mathbf{c}(t), w(t))=\lim _{\tau \rightarrow \infty}(\widetilde{\mathbf{c}}(\tau), \widetilde{w}(\tau))
$$

Step 7 We now show that any solution of (3.79) with initial conditions in $\mathcal{B}_{\eta} \cap \mathcal{T}^{\prime}$ converges to $\left(\mathbf{c}^{*}, 0\right)$ if $\eta$ is taken small enough. This will conclude the proof.

Let $\mathbf{c}(\tau), w(\tau)$ be a solution to (3.79). Then, by Lemma 3.6, we have:

$$
\frac{d}{d \tau} J(\mathbf{c}, w)=-2 \sum_{k=1}^{N}\left(\frac{\rho_{k}}{\sqrt{c_{k}}}-\zeta \pi_{\mathrm{w}} \sqrt{c_{k}}\right)^{2}-2 A w\left(\zeta \pi_{\mathrm{w}}\right)^{2} \equiv \Psi(\mathbf{c}, w)
$$

Since we have removed one factor of $w$ in (3.79) compared with (3.57), one factor of $w$ is removed accordingly from the right hand side of (3.37). 
First, we take $r>0$ small enough so that $J>0$ and $\Psi>0$ for $\overline{\mathcal{B}_{r} \cap \mathcal{T}^{\prime}} \backslash\left\{\left(\mathbf{c}^{*}, 0\right)\right\}$. By (3.73), this is possible for $J$. Consider $\Psi$. If $w>0$, the condition $\Psi=0$ if and only if $\rho_{k}=\pi_{\mathrm{w}}=0$. This is equivalent to the condition for (3.57) to have a steady state in $\mathcal{T}$. By Proposition 3.1, such a point does not exist if $f_{\min }=0$. When $w=0$, $\Psi(\mathbf{c}, 0)=0$ if and only if:

$$
\begin{aligned}
\widehat{L}(\boldsymbol{\gamma}+\mathbf{q})-\zeta \pi_{\mathrm{w}}^{0} \mathbf{c} & =0, \\
\sum_{k=1}^{N} z_{k} c_{k} & =0,
\end{aligned}
$$

where $\pi_{\mathrm{w}}^{0}$ was given in (3.69). It is clear that the point $\mathbf{c}=\mathbf{c}^{*}$ satisfies the above. We show that $\mathbf{c}=\mathbf{c}^{*}$ is the only point that satisfies both (3.85) and (3.86) in a neighborhood of $\mathbf{c}=\mathbf{c}^{*}$ in $\mathbb{R}^{N}$. We use the implicit function theorem. Compute the Jacobian matrix of the left hand side with respect to $\mathbf{c}$ and evaluate this at $\mathbf{c}^{*}$ :

$$
B_{k l}^{*}=\widehat{L}_{k l} \frac{1}{c_{l}^{*}}+\zeta c_{k}^{*} .
$$

Here, $B_{k l}^{*}$ is the $k l$ entry of the $N \times N$ Jacobian matrix $B^{*}$. The rank of $B^{*}$ is the same as the rank of $\widetilde{B}^{*}$ whose $k l$ entry is given by:

$$
\widetilde{B}_{k l}^{*}=\widehat{L}_{k l}+\zeta c_{k}^{*} c_{l}^{*}
$$

Since $\widehat{L}$ is symmetric positive semidefinite with rank $N-1$ (see proof of Proposition 3.4) and $\zeta>0, \widetilde{B}^{*}$, and thus $B^{*}$ is at least rank $N-1$. It is easily checked that $\mathbf{z}_{c}=\left(z_{1} c_{1}^{*}, \ldots, z_{N} c_{N}^{*}\right)^{T}$ is an eigenvector of $B^{*}$ with 0 eigenvalue. Therefore, all the points that satisfy (3.85) near $\mathbf{c}=\mathbf{c}^{*}$ lie on a one-dimensional manifold in $\mathbb{R}^{N}$ that is tangent to $\mathbf{z}_{c}$ at $\mathbf{c}=\mathbf{c}^{*}$. Since $\left\langle\mathbf{z}, \mathbf{z}_{c}\right\rangle_{\mathbb{R}^{N}} \neq 0$, the only common point between this one dimensional manifold and the hyperplane (3.86) is $\mathbf{c}=\mathbf{c}^{*}$.

Define the set:

$$
\mathcal{D}_{\delta}=\left\{(\mathbf{c}, v) \in \mathcal{B}_{r} \cap \mathcal{T}^{\prime} \mid J(\mathbf{c}, v)<\delta\right\} .
$$

Take $\delta=\delta_{0}$ small enough so that $\overline{\mathcal{D}_{\delta_{0}}} \subset \mathcal{B}_{r}$. It is clear by (3.84) that any solution in $\mathcal{D}_{\delta_{0}}$ stays within this set. Choose $\eta$ small enough so that $\mathcal{B}_{\eta} \cap \mathcal{T}^{\prime} \subset \mathcal{D}_{\delta_{0}}$. This is clearly possible since $J$ is a non-negative continuous function on $\mathcal{B}_{r} \cap \mathcal{T}^{\prime}$ which is 0 only at $(\mathbf{c}, w)=\left(\mathbf{c}^{*}, 0\right)$. Take any $\epsilon<\eta$. We may choose a $\delta_{1}>0$ so that $\mathcal{D}_{\delta_{1}} \subset \mathcal{B}_{\epsilon}$. Given that $\Psi>0$ on $\overline{\mathcal{D}_{\delta_{0}} \backslash \mathcal{D}_{\delta_{1}}}$, any solution in $\mathcal{B}_{\eta} \subset \mathcal{D}_{\delta_{0}}$ will be in $\mathcal{B}_{\epsilon} \supset \mathcal{D}_{\delta_{1}}$ in finite time.

In the case of $f_{\min }>0$, we do not have a statement on the limiting value of $\mathbf{c}(t)$ as $t \rightarrow \infty$. The limit point $(\mathbf{c}, w)=\left(\mathbf{c}^{*}, 0\right)$ may well exist and the limiting value

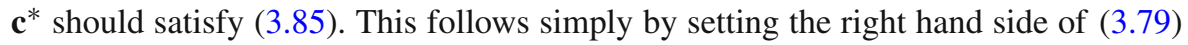
to 0 . If there is only one such point, it should be possible to show, with the aid of the 
Lyapunov function $J$, that this is the single limit point to which all solutions converge. This uniqueness, however, is not clear.

Even though $v(t) \rightarrow \infty$ as $t \rightarrow \infty$ regardless of whether $f_{\min }>0$ or $f_{\min }=0$, the rate at which $v(t)$ grows is different. When $f_{\min }>0$, we see, by combining (3.65) and (3.75) and the definition of $w_{+}(M)$ that $v(t)$ grows at most linearly with time and faster than any power $t^{\alpha}, \alpha<1$. When $f_{\min }=0$, we expect the growth of cell volume to scale like $t^{1 / 2}$, as can be seen by taking the special case $\mathbf{p}=0$ with initial conditions $\mathbf{c}=\mathbf{c}^{\mathrm{e}}$.

\subsection{Epithelial models}

We briefly consider a simple epithelial model. Suppose we have a single layer of epithelial cells which separate the serosal and mucosal sides. The concentrations of electrolytes in the serosal and mucosal solutions are assumed constant. Let these concentrations be denoted $c_{k}^{\mathrm{s}}$ and $c_{k}^{\mathrm{m}}$, respectively. The voltages in the mucosal and serosal sides are also fixed at $\phi^{\mathrm{s}}$ and $\phi^{\mathrm{m}}$. We can write down the following model for electrolyte and water balance for an epithelial cell in this layer:

$$
\begin{aligned}
\frac{d(v \mathbf{c})}{d t} & =-L_{\mathrm{m}} \boldsymbol{\mu}^{\mathrm{m}}-L_{\mathrm{s}} \boldsymbol{\mu}^{\mathrm{s}}-\mathbf{p}^{\mathrm{m}}-\mathbf{p}^{\mathrm{s}}, \\
\frac{d v}{d t} & =-\zeta_{\mathrm{m}} \pi_{\mathrm{w}}^{\mathrm{m}}-\zeta_{\mathrm{s}} \pi_{\mathrm{w}}^{\mathrm{s}}, \\
\sum_{k=1}^{N} z_{k} c_{k}+\frac{z A}{v} & =\sum_{k=1}^{N} z_{k} c_{k}^{\mathrm{m}}=\sum_{k=1}^{N} z_{k} c_{k}^{\mathrm{s}}=0 .
\end{aligned}
$$

The definition of the cellular variables $\mathbf{c}$ and $v$ are the same as before. In the above, $L_{\mathrm{m}, \mathrm{s}}$ are symmetric positive semi-definite matrices and $\mathbf{p}^{\mathrm{m}, \mathrm{s}}$ are the vector of active currents residing on the mucosal and serosal membrane, respectively, which we assume constant. The chemical potentials $\boldsymbol{\mu}^{\mathrm{m}, \mathrm{s}}=\left(\mu_{1}^{\mathrm{m}, \mathrm{s}}, \ldots, \mu_{N}^{\mathrm{m}, \mathrm{s}}\right)^{T}$ are given by:

$$
\mu_{k}^{\mathrm{m}, \mathrm{s}}=\ln \left(\frac{c_{k}}{c_{k}^{\mathrm{m}, \mathrm{s}}}\right)+z_{k}\left(\phi-\phi^{\mathrm{m}, \mathrm{s}}\right),
$$

where $\phi$ is the electrostatic potential inside the cell. The osmotic pressure $\pi_{\mathrm{W}}^{\mathrm{m}, \mathrm{s}}$ is given by:

$$
\pi_{\mathrm{w}}^{\mathrm{m}, \mathrm{s}}=\sum_{k=1}^{N} c_{k}^{\mathrm{m}, \mathrm{s}}-\left(\sum_{k=1}^{N} c_{k}+\frac{A}{v}\right)
$$

and the hydraulic permeabilities $\zeta^{\mathrm{m}, \mathrm{s}}$ are non-negative constants.

The above problem is in fact mathematically identical to system (3.1). Let:

$$
\zeta=\zeta_{\mathrm{m}}+\zeta_{\mathrm{s}}, L=L_{\mathrm{m}}+L_{\mathrm{s}}
$$


and suppose that $\zeta>0$ and $L$ is symmetric positive definite. Define:

$$
\begin{aligned}
c_{k}^{\mathrm{e}} & =\zeta^{-1}\left(\zeta_{\mathrm{m}} c_{k}^{\mathrm{m}}+\zeta_{\mathrm{s}} c_{k}^{\mathrm{s}}\right) \\
\mathbf{p} & =\mathbf{p}^{\mathrm{m}}+\mathbf{p}^{\mathrm{s}}-L_{\mathrm{m}} \boldsymbol{\beta}^{\mathrm{m}}-L_{\mathrm{m}} \boldsymbol{\beta}^{\mathrm{s}} \\
\boldsymbol{\beta}^{\mathrm{m}, \mathrm{s}} & =\left(\beta_{1}^{\mathrm{m}, \mathrm{s}}, \ldots, \beta_{N}^{\mathrm{m}, \mathrm{s}}\right)^{T}, \beta_{k}^{\mathrm{m}, \mathrm{s}}=\ln \left(\frac{c_{k}^{\mathrm{m}, \mathrm{s}}}{c_{k}^{\mathrm{e}}}\right)+z_{k} \phi^{\mathrm{m}, \mathrm{s}}
\end{aligned}
$$

Then, the triple (c, $v, \phi)$ satisfies (3.90) if and only if it satisfies (3.1) with $\zeta, L, \mathbf{c}^{\mathrm{e}}=$ $\left(c_{1}^{\mathrm{e}}, \ldots, c_{N}^{\mathrm{e}}\right)^{T}$ and $\mathbf{p}$ prescribed as in (3.93) and (3.94). We thus have the following result.

Theorem 3.8 Consider system (3.90). Suppose $L_{\mathrm{m}}+L_{\mathrm{s}}$ is symmetric positive definite and $\zeta_{\mathrm{m}}+\zeta_{\mathrm{s}}>0$. Define $f_{\min }$ as in (3.5) in which $\mathbf{q}=\left(q_{1}, \ldots, q_{N}\right)=L^{-1} \mathbf{p}$ and $c_{k}^{\mathrm{e}}, L$ and $\mathbf{p}$ are prescribed as in (3.93) and (3.94). If $f_{\min }<0$, the conclusions of Theorem 3.5 hold. If $f_{\min } \geq 0$, the conclusions of Theorem 3.7 hold.

Note that this epithelial model also enjoys the robustness property described after the end of the proof of Theorem 3.5. We may argue that this is advantageous for an epithelial cell, which must withstand large changes in extracellular ionic concentrations.

\section{Results in the general case}

In the previous section, we assumed that the passive transmembrane ionic flux $j_{k}$ is linear in $\boldsymbol{\mu}$. In this section, we establish results that are valid when we only assume conditions (2.24), (2.26) and (2.27) for $j_{k}$ and $j_{\mathrm{w}}$. In particular, this will apply to the case when the Goldman equation (2.3) is used for $j_{k}$. We also relax the assumption that the pump rates $p_{k}$ be constant. We consider the system:

$$
\begin{aligned}
\frac{d}{d t}(v \mathbf{c}) & =-\mathbf{j}(\phi, \boldsymbol{\mu})-\alpha \mathbf{p}(\phi, \boldsymbol{\mu}), \\
0 & =\sum_{k=1}^{N} z_{k} c_{k}+z \frac{A}{v}=\sum_{k=1}^{N} z_{k} c_{k}^{\mathrm{e}}, \\
\frac{d v}{d t} & =-j_{\mathrm{w}}\left(\pi_{\mathrm{w}}\right)
\end{aligned}
$$

The extracellular ionic concentrations $c_{k}^{\mathrm{e}}, k=1, \ldots, N$ and the amount of impermeable organic solute $A$ are positive. We assume that $\mathbf{j}, \mathbf{p}$ and $j_{\mathrm{w}}$ are $C^{1}$ functions of their arguments. The only difference between this and system (2.11) is that we replaced $\mathbf{p}$ (or $p_{k}$ ) in (2.11a) with $\alpha \mathbf{p}$ (or $\alpha p_{k}$ ) where $\alpha$ is a pump strength parameter. We shall find it useful to vary this parameter in the statements to follow. 


\subsection{Solvability}

We first discuss what we mean by a solution to the initial value problem for (4.1). Consider the two constraints (4.1b) and (the dimensionless form of) (2.8), which we reproduce here for convenience:

$$
\begin{aligned}
Q(\mathbf{c}, v) & \equiv \sum_{k=1}^{N} z_{k} c_{k}+\frac{z A}{v}=0, \\
I(\mathbf{c}, \phi, \alpha) & \equiv \sum_{k=1}^{N} z_{k}\left(j_{k}(\mathbf{c}, \phi)+\alpha p_{k}(\mathbf{c}, \phi)\right)=\langle\mathbf{z}, \mathbf{j}+\alpha \mathbf{p}\rangle_{\mathbb{R}^{N}}=0 .
\end{aligned}
$$

Define the following set:

$$
\Gamma_{\alpha}=\left\{\mathbf{y}=(\mathbf{c}, v, \phi) \in \mathbb{R}_{+}^{N} \times \mathbb{R}_{+} \times \mathbb{R} \mid Q(\mathbf{c}, v)=I_{\alpha}(\mathbf{c}, \phi)=0\right\} .
$$

We shall often omit the dependence of $I$ and $\Gamma$ on $\alpha$.

Definition 4.1 Let $\mathbf{y}^{0}=\left(\mathbf{c}^{0}, v^{0}, \phi^{0}\right) \in \Gamma$ where $\mathbf{c}^{0}=\left(c_{1}^{0}, \ldots, c_{N}^{0}\right)^{T}$. Let $\mathbf{c}(t)=$ $\left(c_{1}(t), \ldots, c_{N}(t)\right)^{T}, v(t), t \geq 0$ be $C^{1}$ functions and $\phi(t), t \geq 0$ be a continuous function of $t$. The function $\mathbf{y}(t)=(\mathbf{c}(t), v(t), \phi(t))$ is a solution to (4.1) with initial values $\mathbf{y}^{0}$ if it satisfies (4.1) and $\mathbf{y}(0)=\mathbf{y}^{0}$. The solution may or may not be defined for all positive time.

Since we are solving a differential algebraic system, we must specify initial conditions that satisfy the constraints. Note that we require $\phi(t)$ to be a continuous function of $t$.

We have the following on the solvability of (4.1).

Lemma 4.2 Let $\mathbf{y}_{0}=\left(\mathbf{c}^{0}, v^{0}, \phi^{0}\right) \in \Gamma$ and suppose $\partial I / \partial \phi \neq 0$ at this point. Let $\mathcal{B}_{r} \subset \mathbb{R}^{N+2}$ be the open ball of radius $r$ centered at $\mathbf{y}_{0}$. Then, there is a $K>0$ such that $\mathcal{B}_{K}$ has the following property.

1. The set $\mathcal{B}_{K} \cap \Gamma$ is an $N$-dimensional submanifold of $\mathbb{R}^{N+2}$. There is a $C^{1}$ function $\Phi$ such that any point $\mathbf{y} \in \mathcal{B}_{K} \cap \Gamma$ can be written as $\mathbf{y}=(\mathbf{c}, v, \Phi(\mathbf{c}))$.

2. Take any point $\mathbf{y}^{1}=\left(\mathbf{c}^{1}, v^{1}, \phi^{1}\right) \in \mathcal{B}_{K} \cap \Gamma$. There is a unique solution $\mathbf{y}(t)=$ $(\mathbf{c}(t), v(t), \phi(t))$ to $(4.1)$ with initial values $\mathbf{y}^{1}$ for short times. For short times, $\phi(t)=\Phi(\mathbf{c}(t))$, and thus $\phi(t)$ is a $C^{1}$ function.

Proof of Lemma 4.2 The first item is a straightforward consequence of the implicit function theorem. For the second item, substitute $\phi=\Phi(\mathbf{c})$ into (4.1). Solve this ODE with initial values $\left(\mathbf{c}^{1}, v^{1}\right)$ and let $\mathbf{c}(t)$ and $v(t)$ be the resulting solution. It is clear that $(\mathbf{c}(t), v(t), \Phi(\mathbf{c}(t)))$ is a solution to (4.1) with initial values $\left(\mathbf{c}^{1}, v^{1}, \phi^{1}\right)$ for short times. This solution is the unique solution, since $\phi(t)$ must be continuous and thus, must remain within $\mathcal{B}_{K}$ for short times.

The same statement clearly holds if we replace $\mathcal{B}_{K}$ with a neighborhood of $\mathbf{y}_{0}$. Note that, when $z \neq 0$, any point in $\mathcal{B}_{K} \cap \Gamma$ can be written as $(\mathbf{c}, V(\mathbf{c}), \Phi(\mathbf{c}))$ where $V(\mathbf{c})$ is 
found by solving $Q(\mathbf{c}, v)=0$ for $v$. Thus, when $z \neq 0$, c serves as a local coordinate system on $\mathcal{B}_{K} \cap \Gamma$.

Given the structure conditions on $j_{k}$ we have the following solvability result.

Proposition 4.3 Suppose $j_{k}$ satisfies (2.24) and (2.27). Let

$$
\mathcal{C}_{r}=\left\{\mathbf{y}=(\mathbf{c}, v, \phi) \in \mathbb{R}^{N+2}|| \mathbf{c}-\mathbf{c}^{\mathrm{e}}|<r,| \phi \mid<r\right\},
$$

where $|\cdot|$ for a vector in $\mathbb{R}^{N}$ denotes its Euclidean norm. There are positive constants $K_{\alpha}$ and $K$ with the following properties.

1. Take any $|\alpha|<K_{\alpha}$. The set $\mathcal{C}_{K} \cap \Gamma_{\alpha}$ is an (unbounded) $N$-dimensional submanifold of $\mathbb{R}^{N+2}$ such that any $\mathbf{y} \in \mathcal{C}_{K} \cap \Gamma_{\alpha}$ can be written as $\mathbf{y}=(\mathbf{c}, v, \Phi(\mathbf{c}, \alpha))$ where $\Phi(\mathbf{c}, \alpha)$ is a $C^{1}$ function of $\mathbf{c}$ and $\alpha$.

2. System (4.1) with initial values $\mathbf{y}^{0}=\left(\mathbf{c}^{0}, v^{0}, \phi^{0}\right) \in \mathcal{C}_{K} \cap \Gamma_{\alpha}$ has a unique solution $\mathbf{y}(t)=(\mathbf{c}(t), v(t), \phi(t))$ for short times. For short times, $\phi(t)=\Phi(\mathbf{c}(t), \alpha)$.

Proof To construct the function $\Phi(\mathbf{c}, \alpha)$, we use the implicit function theorem around $\mathbf{c}=\mathbf{c}^{\mathrm{e}}, \phi=0, \alpha=0$ on $I$. Note that:

$$
I\left(\phi=0, \mathbf{c}=\mathbf{c}^{\mathrm{e}}, \alpha=0\right)=\langle\mathbf{z}, \mathbf{j}(\phi=0, \boldsymbol{\mu}=0)\rangle_{\mathbb{R}^{N}}=0
$$

where we used the definition of $\boldsymbol{\mu}$ in the first equality and (2.24) in the second equality. Take the derivative of $I$ with respect to $\phi$ :

$$
\frac{\partial I}{\partial \phi}=\left\langle\mathbf{z}, \frac{\partial \mathbf{j}}{\partial \phi}+\frac{\partial \mathbf{j}}{\partial \boldsymbol{\mu}} \mathbf{z}\right\rangle_{\mathbb{R}^{N}}+\alpha \frac{\partial p}{\partial \phi} .
$$

In the above, $\mathbf{j}$ is viewed as a function of $\phi$ and $\boldsymbol{\mu}$ whereas $\mathbf{p}$ is viewed as a function of $\phi$ and $\mathbf{c}$. We have used the definition of $\boldsymbol{\mu}$ to obtain the second term in the above. We see that

$$
\frac{\partial I}{\partial \phi}\left(\phi=0, \mathbf{c}=\mathbf{c}^{\mathrm{e}}, \alpha=0\right)=\left\langle\mathbf{z}, \frac{\partial \mathbf{j}}{\partial \boldsymbol{\mu}} \mathbf{z}\right\rangle_{\mathbb{R}^{N}}>0 .
$$

where we used (2.25). Positivity follows from (2.27). With (4.6) and (4.8), we can use the implicit function theorem to obtain a $C^{1}$ function $\Phi$ satisfying

$$
I(\Phi(\mathbf{c}, \alpha), \mathbf{c}, \alpha)=0, \quad \Phi\left(\mathbf{c}^{\mathrm{e}}, 0\right)=0
$$

in a neighborhood of $\mathbf{c}=\mathbf{c}^{\mathrm{e}}, \alpha=0$. The rest of the proof is the same as that of Lemma 4.2.

\subsection{Existence of steady states and asymptotic stability}

A point $\mathbf{y}=(\mathbf{c}, v, \phi) \in \Gamma$ is a steady state of (4.1) if the right hand side of (4.1a) and (4.1c) is 0 at that point. We have the following result on the existence of steady states. This should be seen as a generalization of condition (1.3). 
Proposition 4.4 Suppose $j_{k}$ and $j_{\mathrm{w}}$ satisfy (2.24), (2.26) and (2.27). Then, (4.1) has a steady state with $v>0$ for all sufficiently small $\alpha>0$ so long as the following condition is met:

$$
\left.\left\langle\mathbf{c}^{\mathrm{e}},\left(\frac{\partial \mathbf{j}}{\partial \boldsymbol{\mu}}\right)^{-1} \mathbf{p}\right\rangle_{\mathbb{R}^{N}}\right|_{\phi=0, \boldsymbol{\mu}=\mathbf{0}}>0
$$

where $(\partial \mathbf{j} / \partial \boldsymbol{\mu})^{-1}$ is the inverse of the Jacobian matrix $\partial \mathbf{j} / \partial \boldsymbol{\mu}$.

The idea behind this result is the following. System (4.1) possesses an obvious "steady state" when $\alpha=0$ : $\mathbf{c}=\mathbf{c}^{\mathrm{e}}, \phi=0$ and $v=\infty$. If $\alpha$ is positive but small, we expect this steady state to persist. In order for $v$ to be positive as $\alpha$ is perturbed, we need condition (4.10).

Proof of Proposition 4.4 Let $w=1 / v$. Set the right hand side of (4.1) equal to 0. We have:

$$
\begin{aligned}
j_{k}(\phi, \boldsymbol{\mu})+\alpha p_{k}(\phi, \boldsymbol{\mu}) & =0, \quad k=1, \ldots, N, \\
\sum_{k=1}^{N} z_{k} c_{k}+z A w & =0, \\
j_{\mathrm{w}}\left(\pi_{\mathrm{w}}\right) & =0 .
\end{aligned}
$$

View the above as an equation for $\mathbf{c}, \phi$ and $w$. Note that $\mathbf{c}=\mathbf{c}^{\mathrm{e}}, \phi=0, w=0$ is a solution to the above system if $\alpha=0$. To apply the implicit function theorem, we show that the Jacobian matrix with respect to $\mathbf{c}, \phi, w$ is invertible at this point. This is equivalent to showing that the only solution to the following linear equation for $\widehat{\mathbf{c}}=\left(\widehat{c_{1}}, \ldots, \widehat{c_{N}}\right), \widehat{\phi}$ and $\widehat{w}$ is the trivial one.

$$
\begin{aligned}
\left.\sum_{l=1}^{N} \frac{\partial j_{k}}{\partial \mu_{l}}\right|_{\phi=0, \mu=0}\left(\frac{\widehat{c_{l}}}{c_{l}^{\mathrm{e}}}+z_{l} \widehat{\phi}\right) & =0, \quad k=1, \ldots, N, \\
\sum_{k=1}^{N} z_{k} \widehat{c_{k}}+z A \widehat{w} & =0, \\
\left.\frac{\partial j_{\mathrm{w}}}{\partial \pi_{\mathrm{w}}}\right|_{\pi_{\mathrm{w}}=0}\left(\sum_{k=1}^{N} \widehat{c_{k}}+A \widehat{w}\right) & =0,
\end{aligned}
$$

where we used (2.24) (and its consequence (2.25)) to obtain (4.12). Equation (4.12) together with condition (2.27) and (4.14) together with condition (2.26) gives:

$$
\frac{\widehat{c_{k}}}{c_{k}^{\mathrm{e}}}+z_{k} \widehat{\phi}=0, \quad \sum_{k=1}^{N} \widehat{c_{k}}+A \widehat{w}=0
$$


where the first equation is holds for all $k$. Using (4.15) to eliminate $\widehat{w}$ and $\widehat{c_{k}}$ from (4.13), we have:

$$
\sum_{k=1}^{N}\left(z_{k}-z\right) z_{k} c_{k}^{\mathrm{e}} \widehat{\phi}=\sum_{k=1}^{N} z_{k}^{2} c_{k}^{\mathrm{e}} \widehat{\phi}=0
$$

where we used (4.1b) in the first equality. Since $c_{k}^{\mathrm{e}}$ is positive and at least one of $z_{k} \neq 0$, we see that $\widehat{\phi}=0$. From (4.15), we see that $c_{k}=0$ for all $k$ and $\widehat{w}=0$ since $A>0$. We can thus invoke the implicit function theorem to conclude that we have a solution $\mathbf{c}(\alpha), \phi(\alpha)$ and $w(\alpha)$ to (4.11) when $\alpha$ is close to 0 . To ensure that $w$ (or equivalently, $v$ ) is positive for small $\alpha>0$, we compute:

$$
\left.\frac{d w}{d \alpha}\right|_{\alpha=0}=\left.A^{-1}\left\langle\mathbf{c}^{\mathrm{e}},\left(\frac{\partial \mathbf{j}}{\partial \boldsymbol{\mu}}\right)^{-1} \mathbf{p}\right\rangle_{\mathbb{R}^{N}}\right|_{\phi=0, \boldsymbol{\mu}=\mathbf{0}} .
$$

Since $w=0$ at $\alpha=0$, condition (4.10) will ensure that the $v$ is positive for $\alpha$ small and positive.

We may also compute $d \phi / d \alpha$ :

$$
\begin{aligned}
\left.\frac{d \phi}{d \alpha}\right|_{\alpha=0} & =\left.\left(\sum_{k=1}^{N} z_{k}^{2} c_{k}^{\mathrm{e}}\right)^{-1}\left\langle\left(z \mathbf{c}^{\mathrm{e}}-\mathbf{z}_{c}\right),\left(\frac{\partial \mathbf{j}}{\partial \boldsymbol{\mu}}\right)^{-1} \mathbf{p}\right\rangle_{\mathbb{R}^{N}}\right|_{\phi=0, \boldsymbol{\mu}=\mathbf{0}}, \\
\mathbf{z}_{c} & =\left(z_{1} c_{1}^{\mathrm{e}}, \ldots, z_{N} c_{N}^{\mathrm{e}}\right) .
\end{aligned}
$$

Given (4.10), this shows that the sign of $\phi$ is the same as the sign of $z$ if $|z|$ is large enough. In physiological situations, $z$ is large and negative, and thus we will have a negative membrane potential.

Condition (4.10) applied to (1.1) yields:

$$
\frac{3\left[\mathrm{Na}^{+}\right]_{\mathrm{e}}}{g_{\mathrm{Na}}}-\frac{2\left[\mathrm{~K}^{+}\right]_{\mathrm{e}}}{g_{\mathrm{K}}}>0,
$$

thus reproducing condition (1.3). It is interesting that we do not have any restriction on $z$ for this to be true. Given (4.17), the cell volume will be small if (4.10) is large. Expression (4.19) is indeed large: for a typical cell, $\left[\mathrm{Na}^{+}\right]_{\mathrm{e}} \gg\left[\mathrm{K}^{+}\right]_{\mathrm{e}}$ and $g_{\mathrm{Na}} \ll g_{\mathrm{K}}$. We now turn to the question of stability of steady states.

Definition 4.5 Suppose $\mathbf{y}^{*}=\left(\mathbf{c}^{*}, v^{*}, \phi^{*}\right) \in \Gamma$ is a steady state of (4.1). Let $\mathcal{B}_{r}$ be the open ball of radius $r$ centered at $\mathbf{y}^{*}$. The steady state $\mathbf{y}^{*}$ is stable if the following is true. For any small enough $\epsilon>0$, there exists a $\delta>0$ with the following property. Choose any $\mathbf{y}^{0} \in \mathcal{B}_{\delta} \cap \Gamma$. Then, the solution(s) $\mathbf{y}(t)$ to system (4.1) with initial value $\mathbf{y}^{0}$ is defined for all positive time and satisfies $\mathbf{y}(t) \in \mathcal{B}_{\epsilon}$. The steady state is asymptotically stable if it is stable and all solutions with initial data $\mathbf{y}^{0} \in \Gamma$ sufficiently close to $\mathbf{y}^{*}$ approach $\mathbf{y}^{*}$ as $t \rightarrow \infty$. 
Since any solution lies on $\Gamma$, we may replace $\mathcal{B}_{\epsilon}$ with $\mathcal{B}_{\epsilon} \cap \Gamma$. The only difference between the usual definition of stability and the one above is that the initial data must lie on $\Gamma$. If $\partial I / \partial \phi \neq 0$ at $\mathbf{y}^{*}=\left(\mathbf{c}^{*}, v^{*}, \phi^{*}\right)$ and $z \neq 0$, then, by the remark after the proof of Lemma 4.2, system (4.1) can locally be written as an ODE for c only. The above definition of (asymptotic) stability is then equivalent to the (asymptotic) stability of $\mathbf{c}^{*}$ for this ODE system.

Let $\mathbf{c}^{*}=\left(c_{1}^{*}, \ldots, c_{N}^{*}\right)^{T}, v^{*}, \phi^{*}$ be a steady state of (4.1). Define the following quantities:

$$
\begin{aligned}
\widehat{\phi} & =\phi-\phi^{*} \\
\widehat{\boldsymbol{\gamma}} & =\left(\widehat{\gamma}_{1}, \ldots, \widehat{\gamma}_{N}\right)^{T}, \widehat{\gamma}_{k}=\gamma_{k}-\gamma_{k}^{*}=\ln \left(\frac{c_{k}}{c_{k}^{*}}\right) \\
\widehat{\boldsymbol{\mu}} & =\left(\widehat{\mu}_{1}, \ldots, \widehat{\mu}_{N}\right)^{T}, \widehat{\mu}_{k}=\mu_{k}-\mu_{k}^{*} \equiv \widehat{\gamma}_{k}+z_{k} \widehat{\phi}, \\
\widehat{\pi}_{\mathrm{w}} & =\pi_{\mathrm{w}}-\pi_{\mathrm{w}}^{*} \equiv \sum_{k=1}^{N} c_{k}^{*}+\frac{A}{v^{*}}-\left(\sum_{k=1}^{N} c_{k}+\frac{A}{v}\right) .
\end{aligned}
$$

If $j_{\mathrm{w}}$ satisfies (2.23), $j_{\mathrm{w}}\left(\pi_{\mathrm{w}}\right)=0$ if and only if $\pi_{\mathrm{w}}=0$ and thus $\pi_{\mathrm{w}}^{*}=0$. In this case, $\widehat{\pi}_{\mathrm{w}}=\pi_{\mathrm{w}}$.

Let $\widehat{G}$ be free energy with respect to the steady state defined in (3.21). We have the following analogue of Proposition 2.1 or Lemma 3.2.

Lemma 4.6 Suppose $\mathbf{c}^{*}=\left(c_{1}^{*}, \ldots, c_{N}^{*}\right)^{T}, \phi^{*}, v^{*}$ is a steady state of (4.1). Then, we have:

$$
\frac{d \widehat{G}}{d t}=-\sum_{k=1}^{N} \widehat{\mu}_{k}\left(\widehat{j}_{k}+\alpha \widehat{p}_{k}\right)-\widehat{\pi}_{\mathrm{w}} j_{\mathrm{w}}
$$

where $\widehat{j_{k}}=j_{k}-j_{k}^{*}, \widehat{p}_{k}=p_{k}-p_{k}^{*}$ and $j_{k}^{*}, p_{k}^{*}$ are the passive and active fluxes evaluated at the steady state.

Proof Rewrite (4.1a) as follows:

$$
\frac{\partial\left(v c_{k}\right)}{\partial t}=-\left(j_{k}+\alpha p_{k}\right)+\left(j_{k}^{*}+\alpha p_{k}^{*}\right)=-\sum_{k=1}^{N} \widehat{\mu}_{k}\left(\widehat{j}_{k}+\alpha \widehat{p}_{k}\right),
$$

where we used $j_{k}^{*}+\alpha p_{k}^{*}=0$. Note that, $j_{\mathrm{w}}^{*}$, the water flux at steady state, is equal to 0 . Thus $\widehat{j}_{\mathrm{w}} \equiv j_{\mathrm{w}}-j_{\mathrm{w}}^{*}=j_{\mathrm{w}}$. The rest of the proof is the same as Proposition 2.1.

If we apply the above lemma to system (3.1), this is nothing other than (3.22). The next Lemma gives us a sufficient condition for asymptotic stability in terms of $\widehat{G}$.

Lemma 4.7 Let $\mathbf{y}^{*}=\left(\mathbf{c}^{*}, v^{*}, \phi^{*}\right), \mathbf{c}^{*}=\left(c_{1}^{*}, \ldots, c_{N}^{*}\right)^{T}$ be a steady state of $(4.1)$. Suppose there is neighborhood $\mathcal{U} \subset \mathbb{R}^{N+2}$ of $\mathbf{y}^{*}$ such that $\mathcal{U} \cap \Gamma$ is an N-dimensional submanifold in which any point $\mathbf{y} \in \mathcal{U} \cap \Gamma$ can be written as $\mathbf{y}=(\mathbf{c}, v, \Phi(\mathbf{c}))$ 
where $\Phi$ is a $C^{1}$ function of $\mathbf{c}$. Suppose any solution $\mathbf{y}(t)=(\mathbf{c}(t), v(t), \phi(t))$ in $\mathcal{U}($ or equivalently, in $\mathcal{U} \cap \Gamma$ ) satisfies:

$$
\frac{d \widehat{G}}{d t} \leq-K_{*}\left(|\widehat{\mu}|^{2}+\left|\widehat{\pi}_{\mathrm{W}}\right|^{2}\right)
$$

for some positive constant $K_{*}>0$. Then the steady state is asymptotically stable and the approach to the steady state is exponential in time.

Proof As in the proof of Proposition 3.4, we will find it convenient to use the variables $\mathbf{a}=\left(a_{1}, \ldots, a_{N}\right)^{T}=v \mathbf{c}, v$ and $\phi$ rather than $\mathbf{c}, v$ and $\phi$. We shall continue to use the symbols $\mathcal{U}, \Gamma$ to denote the corresponding sets in the new coordinates. View $\widehat{G}$ as a function of $\mathbf{a}$ and $v$. Note first that:

$$
\begin{aligned}
\widehat{G}\left(\mathbf{a}^{*}, v^{*}\right) & =0, \\
\left.\frac{\partial \widehat{G}}{\partial a_{k}}\right|_{\mathbf{a}=\mathbf{a}^{*}, v=v^{*}} & =\left.\left(\gamma_{k}-\gamma_{k}^{*}\right)\right|_{\mathbf{c}=\mathbf{c}^{*}}=0, \\
\left.\frac{\partial \widehat{G}}{\partial v}\right|_{\mathbf{a}=\mathbf{a}^{*}, v=v^{*}} & =\left.\pi_{\mathrm{w}}\right|_{\mathbf{c}=\mathbf{c}^{*}, v=v^{*}}=0,
\end{aligned}
$$

where $\mathbf{a}^{*}=\left(a_{1}^{*}, \ldots, a_{N}^{*}\right)^{T}=v^{*} \mathbf{c}^{*}$. By Lemma $3.3, \widehat{G}$ is a globally convex function on $(\mathbf{a}, v) \in \mathbb{R}_{+}^{N+1}$. The point $(\mathbf{a}, v)=\left(\mathbf{a}^{*}, v^{*}\right)$ is thus the global minimizer of $\widehat{G}$. The positive definiteness of the Hessian matrix of $\widehat{G}$ implies that there is a neighborhood $\mathcal{N} \subset \mathbb{R}^{N+1}$ of $\left(\mathbf{a}^{*}, v^{*}\right)$ where

$$
K_{G}^{-1}\left(\left|\mathbf{a}-\mathbf{a}^{*}\right|^{2}+\left|v-v^{*}\right|^{2}\right) \leq \widehat{G}(\mathbf{a}, v) \leq K_{G}\left(\left|\mathbf{a}-\mathbf{a}^{*}\right|^{2}+\left|v-v^{*}\right|^{2}\right)
$$

for some positive constant $K_{G}$.

Now, consider $\mathbb{R}^{N+2}$ with the coordinates $(\mathbf{a}, v, \phi)$. Define $Q=\sum_{k=1}^{N} z_{k}\left(a_{k}-a_{k}^{*}\right)$. This $Q$ is the same as the $Q$ in (4.2) except that it is written in terms of a. We claim that, in the vicinity of $\left(\mathbf{a}^{*}, v^{*}, \phi^{*}\right)$ in $\mathbb{R}^{N+2}$, the set of variables $\left(\widehat{\boldsymbol{\mu}}, \widehat{\pi}_{\mathrm{w}}, Q\right)$ defines a coordinate system. It is easily seen that the variables $(\mathbf{c}, v, \phi)$ defines a coordinate system. We thus consider the coordinate change from $\left(\widehat{\boldsymbol{\mu}}, \widehat{\pi}_{\mathrm{w}}, Q\right)$ to $(\mathbf{c}, v, \phi)$. The Jacobian matrix between these two sets of variables at $(\mathbf{c}, v, \phi)=\left(\mathbf{c}^{*}, v^{*}, \phi^{*}\right)$ is non-singular. This computation is almost the same as the computation in the proof of Proposition 4.4, so we omit the details. The claim follows by the implicit function theorem. There is therefore a neighborhood $\mathcal{V} \subset \mathbb{R}^{N+2}$ of $\left(\mathbf{a}^{*}, v^{*}, \phi^{*}\right)$ in which the following inequality holds:

$$
K_{\mu}\left(\left|\mathbf{a}-\mathbf{a}^{*}\right|^{2}+\left|v-v^{*}\right|^{2}+\left|\phi-\phi^{*}\right|^{2}\right) \leq|\widehat{\boldsymbol{\mu}}|^{2}+\left|\widehat{\pi}_{\mathrm{w}}\right|^{2}+|Q|^{2}
$$

where $K_{\mu}$ is a positive constant. Any solution to (4.1) satisfies $Q=0$ (see (4.2)). Thus, we have: 


$$
K_{\mu}\left(\left|\mathbf{a}-\mathbf{a}^{*}\right|^{2}+\left|v-v^{*}\right|^{2}\right) \leq|\widehat{\boldsymbol{\mu}}|^{2}+\left|\pi_{\mathrm{w}}\right|^{2}
$$

for any solution in $\mathcal{V}$.

Choose a neighborhood $\mathcal{M} \subset \mathbb{R}^{N+1}$ of $\left(\mathbf{a}^{*}, v^{*}\right)$ such that $(\mathbf{a}, v, \Phi(\mathbf{a} / v)) \in \mathcal{U} \cap \mathcal{V}$ for all $(\mathbf{a}, v) \in \mathcal{M}$. Consider the following set:

$$
\mathcal{G}=\left\{(\mathbf{a}, v) \in \mathbb{R}^{N+1} \mid \widehat{G}(\mathbf{a}, v)<M_{G}, M_{G}>0\right\}
$$

Since $\widehat{G}(\mathbf{a}, v)$ is a convex function such that $G\left(\mathbf{a}^{*}, v^{*}\right)=0, \mathcal{G}$ is an open neighborhood of $\left(\mathbf{a}^{*}, v^{*}\right)$, and can be made arbitrarily small by making $M_{G}$ small. Take $M_{G}$ so small that $\mathcal{G} \subset \mathcal{N} \cap \mathcal{M}$. Consider the following open neighborhood of $\left(\mathbf{a}^{*}, v^{*}, \phi^{*}\right)$ :

$$
\mathcal{W}=\left\{\mathbf{y}=(\mathbf{a}, v, \phi) \in \mathbb{R}^{N+2} \mid(\mathbf{a}, v) \in \mathcal{G}, \mathbf{y} \in \mathcal{U} \cap \mathcal{V}\right\}
$$

Any solution in $\mathcal{W}$, by definition, belongs to $\mathcal{W} \cap \Gamma$. For any such solution, we have:

$$
\begin{aligned}
\frac{d \widehat{G}}{d t} & \leq-K_{*}\left(|\widehat{\boldsymbol{\mu}}|^{2}+\left|\widehat{\pi}_{\mathrm{w}}\right|^{2}\right) \\
& \leq-K_{*} K_{\mu}\left(\left|\mathbf{a}-\mathbf{a}^{*}\right|^{2}+\left|v-v^{*}\right|^{2}\right) \leq-\frac{K_{*} K_{\mu}}{K_{G}} \widehat{G}
\end{aligned}
$$

where we used (4.23) in the first inequality, (4.27) in the second inequality and (4.25) in the third inequality. Solving the above differential inequality, we have:

$$
\widehat{G} \leq M_{G} \exp \left(-K_{*} K_{\mu} t / K_{G}\right), t \geq 0
$$

We thus see that $\mathcal{W} \cap \Gamma$ is a positively invariant set, and thus, all solutions starting from $\mathcal{W} \cap \Gamma$ are defined for all time. By $(4.25),(\mathbf{a}(t), v(t))$ approaches $\left(\mathbf{a}^{*}, v^{*}\right)$ exponentially in time. Since $(\mathcal{W} \cap \Gamma) \subset(\mathcal{U} \cap \Gamma), \phi=\Phi(\mathbf{a}(t) / v(t))$. Since $\Phi$ is a $C^{1}$ function, $\phi(t)$ also approaches $\phi^{*}$ exponentially in time.

We are now ready to state the main result of this section.

Theorem 4.8 Suppose $j_{k}$ and $j_{\mathrm{w}}$ satisfy (2.24), (2.27) and (2.26) and $j_{k}, p_{k}$ and $c_{k}^{\mathrm{e}}$ satisfy (4.10). For all sufficiently small $\alpha>0$, the steady states found in Proposition 4.4 are asymptotically stable. The approach to steady state is exponential in time.

In Proposition 3.4, we used the symmetry condition of (2.27) to show that the eigenvalues of the linearized matrix around steady state are all real. Here, we cannot prove such a statement. In fact, the proof to follow goes through even if we assume (2.28) instead of (2.27).

We also point out that, unlike Proposition 3.4 or Theorem 3.5, we can only draw conclusions when the pump rate is small ( $\alpha$ is small). One may wonder whether it may be possible to generalize Theorem 4.8 to the case when the pump rate is not necessarily small. For this, one would clearly need a condition stronger than (2.26) or 
(2.27). One natural idea is to require that $j_{k}$ and $j_{\mathrm{w}}$ satisfy (2.26) and (2.27) not only at $\pi_{\mathrm{w}}=0$ and $\mu=0$ but at any arbitrary value of $\pi_{\mathrm{w}}$ and $\mu$ :

$$
\begin{aligned}
& \frac{\partial j_{\mathrm{w}}}{\partial \pi_{\mathrm{w}}}\left(\pi_{\mathrm{w}}\right)>0 \text { for all } \pi_{\mathrm{w}}, \\
& \frac{\partial \mathbf{j}}{\partial \boldsymbol{\mu}}(\phi, \boldsymbol{\mu}) \text { is a positive definite matrix for all } \phi \text { and } \boldsymbol{\mu} \text {. }
\end{aligned}
$$

This stronger condition is indeed satisfied by the Goldman equation (2.3) (and trivially by (2.2)). Let us assume the pump rates $\alpha p_{k}$ are constant. A natural conjecture may be that if $j_{\mathrm{W}}$ and $j_{k}$ satisfy (4.32), any steady state of (4.1) is stable (whether or not the pump rate is small). This statement is true provided that $j_{k}$ is only a function of $\boldsymbol{\mu}$ and not a function of $\phi$. In the case of (2.2) or (3.1), $j_{k}$ is indeed only a function of $\boldsymbol{\mu}$. Unfortunately, (2.3) is a function of both $\boldsymbol{\mu}$ and $\phi$. The danger when $j_{k}$ depends on $\phi$ independently of $\boldsymbol{\mu}$ is that $\partial j_{k} / \partial \phi$ may adversely affect the stability properties imparted by condition (4.32).

When the pump strength is small ( $\alpha$ small), $\partial j_{k} / \partial \phi$ is small provided $\alpha$ is small thanks to condition (2.25). This is one of the key observations that we will use in the proof to follow.

Proof of Theorem 4.8 Let $\mathbf{y}^{*}=\left(\mathbf{c}^{*}, v^{*}, \phi^{*}\right)$ be the steady state found in Proposition 4.4. As $\alpha>0$ is made small, $\mathbf{y}^{*} \in \mathcal{C}_{K} \cap \Gamma$ defined in Proposition 4.3. We shall henceforth assume that $\mathbf{y}^{*} \in \mathcal{C}_{K} \cap \Gamma$.

By Proposition 4.3, Lemmas 4.6 and 4.7 , it is sufficient to show that, for sufficiently small $\alpha$, there is a neighborhood $\mathcal{U} \subset \mathbb{R}^{N+2}$ of $\left(\mathbf{c}^{*}, v^{*}, \phi^{*}\right)$ such that any $\mathbf{y}=(\mathbf{c}, v, \phi) \in \mathcal{U} \cap \Gamma$ satisfies the following inequality:

$$
K_{*}\left(|\widehat{\mu}|^{2}+\left|\widehat{\pi}_{\mathrm{w}}\right|^{2}\right) \leq \sum_{k=1}^{N} \widehat{\mu}_{k}\left(\widehat{j}_{k}+\alpha \widehat{p}_{k}\right)+\widehat{\pi}_{\mathrm{w}} j_{\mathrm{w}} \equiv J
$$

for some $K_{*}>0$. The neighborhood $\mathcal{U}$ may depend on $\alpha$.

Recall from the proof of Lemma 4.7 that $\left(\widehat{\boldsymbol{\mu}}, \pi_{\mathrm{w}}, Q\right)$ defines a coordinate system in the vicinity $\mathcal{N}$ of $\left(\mathbf{c}^{*}, v^{*}, \phi^{*}\right)$. Note that the point $\left(\mathbf{c}^{*}, v^{*}, \phi^{*}\right)$ is the origin in the coordinate system $\left(\widehat{\boldsymbol{\mu}}, \pi_{\mathrm{w}}, Q\right)$. Let:

$$
\mathcal{D}_{r}=\left\{\left.(\mathbf{c}, v, \phi) \in \mathcal{N}|| \widehat{\boldsymbol{\mu}}\right|^{2}+\widehat{\pi}_{\mathrm{W}}^{2}+Q^{2}<r^{2}, r>0\right\}
$$

and take $r$ small enough so that $\mathcal{D}_{r} \subset \mathcal{C}_{K}$. Define the set:

$$
\Gamma_{Q}=\left\{\mathbf{y}=(\mathbf{c}, v, \phi) \in \mathbb{R}_{+}^{N} \times \mathbb{R}_{+} \times \mathbb{R} \mid Q(\mathbf{c}, v)=0\right\}
$$

Since $\Gamma \subset \Gamma_{Q}$, it is clearly sufficient if we can find a small enough $r>0$ such that (4.33) holds for any point in $\mathcal{D}_{r} \cap \Gamma_{Q}$.

We can write $\widehat{\phi}=\phi-\phi^{*}$ as a function of $\widehat{\mu}, \pi_{\mathrm{w}}$ and $Q$ in $\mathcal{D}_{r}$. In particular, on $\mathcal{D}_{r} \cap \Gamma_{Q}, \widehat{\phi}$ is a function of $\widehat{\boldsymbol{\mu}}$ and $\widehat{\pi}_{\mathrm{w}}$ only. Call this function $\widehat{\phi}=\varphi\left(\widehat{\boldsymbol{\mu}}, \widehat{\pi}_{\mathrm{w}}\right)$. 
The function $\varphi$ satisfies:

$$
0=\sum_{k=1}^{N}\left(z_{k}-z\right) c_{k}^{*}\left(\exp \left(\widehat{\mu}_{k}-z_{k} \varphi\right)-1\right)-z \widehat{\pi}_{\mathrm{w}}
$$

This equation is obtained by expressing $c_{k}$ and $v$ in terms of $\widehat{\mu}_{k}, \widehat{\pi}_{\mathrm{w}}$ and $\widehat{\phi}$ and substituting this into $Q=0$.

Now, take any point $\mathbf{w}=\left(\widehat{\boldsymbol{\mu}}^{1}, \widehat{\pi}_{\mathrm{w}}^{1}, 0\right)=\left(\widehat{\mu}_{1}^{1}, \ldots, \widehat{\mu}_{N}^{1}, \widehat{\pi}_{\mathrm{w}}^{1}, 0\right) \in \mathcal{D}_{r} \cap \Gamma_{Q}$ where we have expressed the point $\mathbf{w}$ using the $\left(\widehat{\boldsymbol{\mu}}, \widehat{\pi}_{\mathrm{w}}, Q\right)$ coordinate system. Let us compute the right hand side of (4.33) at this point.

$$
J\left(\widehat{\boldsymbol{\mu}}^{1}, \widehat{\pi}_{\mathrm{w}}^{1}\right)=\sum_{k=1}^{N} \widehat{\mu}_{k}^{1}\left(\widehat{j}_{k}\left(\varphi\left(\widehat{\boldsymbol{\mu}}^{1}, \widehat{\pi}_{\mathrm{w}}^{1}\right), \widehat{\boldsymbol{\mu}}^{1}\right)+\alpha \widehat{p}_{k}\left(\varphi\left(\widehat{\boldsymbol{\mu}}^{1}, \widehat{\pi}_{\mathrm{w}}^{1}\right), \widehat{\boldsymbol{\mu}}^{1}\right)\right)+\widehat{\pi}_{\mathrm{w}}^{1} j_{\mathrm{w}}\left(\widehat{\pi}_{\mathrm{w}}^{1}\right)
$$

where we took $\widehat{j}_{k}, \widehat{p}_{k}$ as functions of $\widehat{\phi}, \widehat{\boldsymbol{\mu}}$ and $\widehat{j}_{\mathrm{w}}$ as a function of $\widehat{\pi}_{\mathrm{w}}$. For $\widehat{j}_{k}$, we have:

$$
\begin{aligned}
\widehat{j_{k}}\left(\varphi\left(\widehat{\boldsymbol{\mu}}^{1}, \widehat{\pi}_{\mathrm{w}}^{1}\right), \widehat{\boldsymbol{\mu}}^{1}\right)= & \int_{0}^{1} \frac{d}{d s} \widehat{j_{k}}\left(\varphi\left(s \widehat{\boldsymbol{\mu}}^{1}, s \widehat{\pi}_{\mathrm{w}}\right), s \widehat{\boldsymbol{\mu}}^{1}\right) d s \\
= & \sum_{l=1}^{N} \widehat{\mu}_{l}^{1} \int_{0}^{1}\left(\left(\frac{\partial \widehat{j}_{k}}{\partial \widehat{\phi}} \frac{\partial \varphi}{\partial \mu_{l}}+\frac{\partial \widehat{j}_{k}}{\partial \widehat{\mu}_{l}}\right)\left(s \widehat{\boldsymbol{\mu}}^{1}, s \widehat{\pi}_{\mathrm{w}}\right)\right) d s \\
& +\widehat{\pi}_{\mathrm{w}}^{1} \int_{0}^{1}\left(\frac{\partial \widehat{j}_{k}}{\partial \phi} \frac{\partial \varphi}{\partial \widehat{\pi}_{\mathrm{w}}}\left(s \widehat{\boldsymbol{\mu}}^{1}, s \widehat{\pi}_{\mathrm{w}}\right)\right) d s .
\end{aligned}
$$

Performing a similar calculation for $\widehat{p}_{k}$ and $\widehat{j}_{\mathrm{w}}$ and substituting this back into (4.37), we obtain the following expression.

$$
\begin{aligned}
J(\boldsymbol{\psi}) & =\langle\boldsymbol{\psi}, P \boldsymbol{\psi}\rangle_{\mathbb{R}^{N+1}}, \quad \boldsymbol{\psi}=\left(\widehat{\boldsymbol{\mu}}^{1}, \widehat{\pi}_{\mathrm{w}}^{1}\right), \\
P & =\int_{0}^{1}(L+B+C)\left(s \widehat{\boldsymbol{\mu}}^{1}, s \widehat{\pi}_{\mathrm{w}}^{1}\right) d s,
\end{aligned}
$$

where $L, B$ and $C$ are $(N+1) \times(N+1)$ matrix-valued functions defined on $\mathcal{D}_{r} \cap \Gamma_{Q}$, given as follows. Let $L_{k l}, B_{k l}$ and $C_{k l}$ be the $k l$ entries of these matrices.

$$
L_{k l}= \begin{cases}\partial \widehat{j}_{k} / \partial \widehat{\mu}_{l} & \text { if } 1 \leq k, l \leq N \\ \partial \widehat{j}_{\mathrm{w}} / \partial \widehat{\pi}_{w} & \text { if } k=l=N+1 \\ 0 & \text { otherwise }\end{cases}
$$




$$
\begin{aligned}
B_{k l} & = \begin{cases}\left(\partial \widehat{j}_{k} / \partial \widehat{\phi}\right)\left(\partial \varphi / \partial \widehat{\mu}_{l}\right) & \text { if } 1 \leq k, l \leq N, \\
\left(\partial \widehat{j}_{k} / \partial \widehat{\phi}\right)\left(\partial \varphi / \partial \widehat{\pi}_{\mathrm{W}}\right) & \text { if } 1 \leq k \leq N, l=N+1, \\
0 & \text { otherwise, }\end{cases} \\
C_{k l} & = \begin{cases}\alpha\left(\partial \widehat{p}_{k} / \partial \widehat{\mu}_{l}+\left(\partial \widehat{p}_{k} / \partial \widehat{\phi}\right)\left(\partial \varphi / \partial \widehat{\mu}_{l}\right)\right) & \text { if } 1 \leq k, l \leq N, \\
\alpha\left(\partial \widehat{p}_{k} / \partial \widehat{\phi}\right)\left(\partial \varphi / \partial \widehat{\pi}_{\mathrm{W}}\right) & \text { if } 1 \leq k \leq N, l=N+1, \\
0 & \text { otherwise. }\end{cases}
\end{aligned}
$$

To show that (4.33) is valid in $\mathcal{D}_{r} \cap \Gamma_{Q}$, it is sufficient to show that $L+B+C$ is positive definite in $\mathcal{D}_{r} \cap \Gamma_{Q}$ in the sense that:

$$
\langle\mathbf{x},(L+B+C) \mathbf{x}\rangle_{\mathbb{R}^{N+1}} \geq K_{*}|\mathbf{x}|^{2}
$$

for any $\mathbf{x} \in \mathbb{R}^{N+1}$ with a constant $K_{*}>0$ that does not depend on the point in $\mathcal{D}_{r} \cap \Gamma_{Q}$. Since $L, B$ and $C$ are continuous functions on $\mathcal{D}_{r} \cap \Gamma_{Q}$ and we may take $r$ as small as we want, all we have to show is that (4.43) holds at the origin, $\left(\widehat{\boldsymbol{\mu}}, \widehat{\pi}_{\mathrm{w}}, Q\right)=(\mathbf{0}, 0,0)$, or equivalently, at the steady state.

Let $L^{*}, B^{*}$ and $C^{*}$ be the evaluation of the three matrices at steady state. Since the steady state is a function of $\alpha, L^{*}, B^{*}$ and $C^{*}$ are functions of $\alpha$. We will show that (4.43) holds for sufficiently small $\alpha>0$.

First, let us examine the behavior of $\mu^{*}, \pi_{\mathrm{w}}^{*}, \phi^{*}$ as a function of $\alpha$. By Proposition $4.4, \mathbf{c}^{*}, \phi^{*}$ are $C^{1}$ functions of $\alpha$ that approach $\mathbf{c}^{\mathrm{e}}, 0$, respectively, as $\alpha \rightarrow 0$. Therefore, $\boldsymbol{\mu}^{*}$ is a $C^{1}$ function of $\alpha$ that approaches $\mathbf{0}$ as $\alpha \rightarrow 0$. It is clear that $\pi_{\mathrm{w}}^{*}=0$ for any $\alpha$.

The $k l$ entry of the matrix $L^{*}$ is given by:

$$
L_{k l}^{*}= \begin{cases}\left.\left(\partial j_{k} / \partial \mu_{l}\right)\right|_{\phi=\phi^{*}, \mu=\mu^{*}} & \text { if } 1 \leq k, l \leq N, \\ \left.\left(\partial j_{\mathrm{w}} / \partial \pi_{\mathrm{w}}\right)\right|_{\pi_{\mathrm{w}}=0} & \text { if } k=l=N+1, \\ 0 & \text { otherwise. }\end{cases}
$$

Since $\boldsymbol{\mu}^{*} \rightarrow \mathbf{0}$ and $\phi^{*} \rightarrow 0$ as $\alpha \rightarrow 0$, given (2.27) and (2.26), there is a constant $K_{L}>0$ such that:

$$
\left\langle\mathbf{x}, L^{*} \mathbf{x}\right\rangle_{\mathbb{R}^{N+1}} \geq K_{L}|\mathbf{x}|^{2}
$$

for sufficiently small $\alpha>0$.

To examine $B^{*}$ and $C^{*}$, let us first compute $\partial \varphi / \partial \widehat{\mu}_{l}$ and $\partial \varphi / \partial \widehat{\pi}_{\mathrm{w}}$. This can be computed by taking the partial derivatives of (4.36):

$$
\begin{aligned}
& \left.\frac{\partial \varphi}{\partial \widehat{\mu}_{l}}\right|_{\widehat{\boldsymbol{\mu}}=\mathbf{0}, \widehat{\pi}_{\mathrm{w}}=0}=\left(z_{l}-z\right) c_{l}^{*}\left(\sum_{k=1}^{N} z_{k}^{2} c_{k}^{*}+z^{2} \frac{A}{v^{*}}\right)^{-1}, \\
& \left.\frac{\partial \varphi}{\partial \widehat{\pi}_{\mathrm{w}}}\right|_{\widehat{\boldsymbol{\mu}}=\mathbf{0}, \widehat{\pi}_{\mathrm{w}}=0}=-z\left(\sum_{k=1}^{N} z_{k}^{2} c_{k}^{*}+z^{2} \frac{A}{v^{*}}\right)^{-1} .
\end{aligned}
$$


Since $c_{k}^{*} \rightarrow c_{k}^{e}$ and $1 / v^{*} \rightarrow 0$ as $\alpha \rightarrow 0$, we see that both of the above are bounded (and in fact has a definite limit) as $\alpha \rightarrow 0$.

Let us examine $B^{*}$. For $1 \leq k, l \leq N$, the $k l$ entry of the matrix $B^{*}$ is given by:

$$
B_{k l}^{*}=\left.\left(z_{l}-z\right) c_{l}^{*}\left(\sum_{k=1}^{N} z_{k}^{2} c_{k}^{*}+z^{2} \frac{A}{v^{*}}\right)^{-1}\left(\frac{\partial j_{k}}{\partial \phi}\right)\right|_{\phi=\phi^{*}, \mu=\mu^{*}} .
$$

Recall that $\partial j_{k} / \partial \phi=0$ at $\boldsymbol{\mu}=\mathbf{0}$ from (2.25). Since $\phi^{*} \rightarrow 0$ and $\boldsymbol{\mu}^{*} \rightarrow \mathbf{0}$ as $\alpha \rightarrow 0$, by (2.24), we see that $B_{k l}^{*} \rightarrow 0$ as $\alpha \rightarrow 0$. Likewise, $B_{k l}^{*} \rightarrow 0$ as $\alpha \rightarrow 0$ when $1 \leq k \leq N$ and $l=N+1$.

Now, consider $C_{k l}^{*}$, the $k l$ entries of the matrix $C^{*}$. For $1 \leq k, l \leq N$, we have:

$$
C_{k l}^{*}=\left.\alpha\left(\frac{\partial p_{k}}{\partial \mu_{l}}+\left(z_{l}-z\right) c_{l}^{*}\left(\sum_{k=1}^{N} z_{k}^{2} c_{k}^{*}+z^{2} \frac{A}{v^{*}}\right)^{-1} \frac{\partial p_{k}}{\partial \phi}\right)\right|_{\phi=\phi^{*}, \boldsymbol{\mu}=\boldsymbol{\mu}^{*}} .
$$

Given that $\boldsymbol{\mu}^{*} \rightarrow \mathbf{0}, \phi^{*} \rightarrow 0$ as $\alpha \rightarrow 0$, the quantity inside the outer-most parentheses remains bounded as $\alpha \rightarrow 0$. Thus, $C_{k l}^{*} \rightarrow 0$ as $\alpha \rightarrow 0$. The same conclusion holds for $C_{k l}^{*}, 1 \leq k \leq N, l=N+1$.

Since $L^{*}$ satisfies (4.45) from sufficiently small $\alpha$ and $B^{*}$ and $C^{*}$ both tend to the zero matrix as $\alpha \rightarrow 0, L^{*}+B^{*}+C^{*}$ satisfies (4.43) with $K_{*}=K_{L} / 2$ for small enough $\alpha$.

\section{Discussion}

In this paper, we presented what the author believes is the first analytical result on the stability of steady states of pump-leak models. In Sect. 3, we studied the case in which the flux functions are linear in the chemical potential. In the proof of Proposition 3.4, we saw that the system can be seen as a gradient flow of a convex function. This is nothing other than the relaxation law postulated in linear non-equilibrium thermodynamics, $d \mathbf{X} / d t=-L \nabla_{X} G$, where $\mathbf{X}$ is the vector of extensive variable, $L$ is the matrix of transport coefficients and $\nabla_{X} G$ is the gradient of the free energy $G$ with respect to X (Katzir-Katchalsky and Curran 1965; Kjelstrup and Bedeaux 2008). There are two interesting points here. The first point is that this gradient flow is restricted to flow on a submanifold on which electroneutrality holds. The electrostatic potential, as we discussed in the proof of Lemma 3.3 can then be seen as a Lagrange multiplier of this gradient flow. The second point is that we can find a suitable modification of the free energy $(\widetilde{G}$ or $\widehat{G})$ so that our system is a gradient flow even in the presence of active currents. We proved the following results. If condition (3.5) is satisfied, there is a unique steady state that is globally asymptotically stable. If not, the cell volume tends to infinity as time $t \rightarrow \infty$. The system is thus robust to external perturbations in the following sense. Suppose the cell is subject to a change in extracellular concentration or pump rate that stays within the bounds of condition (3.5). The cell will eventually 
approach the new global steady state. We saw that the same conclusions hold for a simple epithelial model, since it could be mapped to the single cell problem.

In Sect. 4, we proved that steady states for pump-leak models are stable so long as the steady state is not too far away from thermodynamic equilibrium. The "stable equilibrium state" is the "state" at which all intracellular ionic concentrations $c_{k}$ are equal to the extracellular concentration $c_{k}^{\mathrm{e}}$ and the cell volume $v$ is infinite. If the pumps work in the "right direction" (in the sense of Proposition 4.4) $v$ can be made finite even with a small pump rate. A biophysical interpretation of Theorem 4.8 is that if the pump rate is sufficiently small, the new steady state of finite volume is still close enough to thermodynamic equilibrium so that the steady state inherits the stability properties of the equilibrium state. It is interesting that stability of thermodynamic equilibrium, which may be considered the "dead" state, confers stability to the "live" state.

The results of Sect. 4, though applicable to general pump-leak models, only asserts the existence of at least one asymptotically stable steady state for small pump rates. To draw analytical conclusions at large pump rates without the linearity assumption of Sect. 3, it is likely that one would need to look at special characteristics of specific pump-leak models. Our current study may thus be seen as complementing computational investigations of stability, in which one is not constrained to small pump rates (Weinstein 1997). We also point out that, for general pump-leak models, we cannot rule out the possibility of multiple steady states or of other non-trivial asymptotic behavior. Indeed, Weinstein (1992) reports an instance in which there are two stable steady states in a non-electrolyte model of epithelial cell volume control.

Many epithelial models include effects not included in model (2.11) or (3.90). Of particular importance is the incorporation of acid-base reactions (Weinstein 1983; Strieter et al. 1990). It is usually assumed that the acid-base reactions are sufficiently fast so that these reactions are in equilibrium. This gives rise to additional algebraic constraints, increasing the co-dimension of the differential algebraic system (Weinstein 2002, 2004; Weinstein and Sontag 2009). It would be interesting to see whether the analysis of this paper can be extended to this case. A starting point for an analytical study of such models will likely be a free energy identity. A potential complication is that the algebraic constraints of acid-base reactions are not linear in the concentrations. This may pose difficulties in extending the global results of Sect. 3. The author hopes to report on such an extension in future work.

Stability of steady states is just a starting point in the study of homeostatic control in epithelial systems. In Weinstein $(2002,2004)$ and Weinstein and Sontag (2009), the authors go beyond stability to study the optimal control of homeostatic parameters by minimizing a quadratic cost function along a relaxation trajectory. We hope that our current study will lead to new insights into such problems.

Free energy dissipation identities similar to (2.13) are present in many models of soft-condensed matter physics (Doi and Edwards 1988; Doi 2009), and can be used as a guiding principle in formulating models in dissipative systems (Eisenberg et al. 2010; Mori et al. 2011). The present work owes much of its inspiration to this body of work. We believe that there is much to be gained by a systematic application of these ideas to the study of physiological systems. We hope that this paper will be a starting point in this direction. 
Acknowledgments This work was inspired by the numerous discussions the author had with Chun Liu and Robert S. Eisenberg at the IMA (Institute for Mathematics and its Applications). This work was supported by NSF Grant DMS-0914963, the Alfred P. Sloan Foundation and the McKnight Foundation.

\section{References}

Armstrong C (2003) The Na/K pump, Cl ion, and osmotic stabilization of cells. Proc Natl Acad Sci USA 100(10): 6257

Benson J, Chicone C, Critser J (2010) A general model for the dynamics of cell volume, global stability, and optimal control. J Math Biol 63:1-21

Boron W, Boulpaep E (2008) Medical physiology, 2nd edn. W.B. Saunders, Philadelphia

Chicone C (1999) Ordinary differential equations with applications. Texts in applied mathematics. Springer, Berlin

Doi M (2009) Gel dynamics. J Phys Soc Jpn 78:052001

Doi M, Edwards S (1988) The theory of polymer dynamics. International series of monographs on physics. Clarendon Press, Oxford

Doi M, See H (1996) Introduction to polymer physics. Oxford University Press, USA

Eisenberg B, Hyon Y, Liu C (2010) Energy variational analysis of ions in water and channels: field theory for primitive models of complex ionic fluids. J Chem Phys 133:104104

Evans D (2009) Osmotic and ionic regulation: cells and animals. CRC Press, West Palm Beach

Fischbarg J, Diecke F (2005) A mathematical model of electrolyte and fluid transport across corneal endothelium. J Membr Biol 203(1):41-56

Fromter E (1974) Electrophysiology and isotonic fluid absorption of proximal tubules of mammalian kidney. In: Thurau K (ed) Kidnery and urinary tract physiology. Butterworths, London pp 1-38

Hernández J (2003) Stability properties of elementary dynamic models of membrane transport. Bull Math Biol 65(1):175-197

Hernández J (2007) A general model for the dynamics of the cell volume. Bull Math Biol 69(5):1631-1648

Hernández JA, Cristina E (1998) Modeling cell volume regulation in nonexcitable cells: the roles of the na+ pump and of cotransport systems. Am J Physiol Cell Physiol 275(4):C1067

Hoffmann E, Lambert I, Pedersen S (2009) Physiology of cell volume regulation in vertebrates. Physiol Rev 89(1):193

Hoppensteadt F, Peskin C (2002) Modeling and simulation in medicine and the life sciences. Springer, Berlin

Jakobsson E (1980) Interactions of cell volume, membrane potential, and membrane transport parameters. Am J Physiol Cell Physiol 238(5):C196

Katzir-Katchalsky A, Curran P (1965) Nonequilibrium thermodynamics in biophysics. Harvard University Press, Cambridge

Keener J, Sneyd J (1998) Mathematical physiology. Springer, New York

Kjelstrup S, Bedeaux D (2008) Non-equilibrium thermodynamics of heterogeneous systems. World Scientific Publishing Co, Hackensack

Larsen E, Sørensen J, Sørensen J (2002) Analysis of the sodium recirculation theory of solute-coupled water transport in small intestine. J Physiol 542(1):33-50

Lew V, Freeman C, Ortiz O, Bookchin R (1991) A mathematical model of the volume, pH, and ion content regulation in reticulocytes. Application to the pathophysiology of sickle cell dehydration. J Clin Investig 87(1):100

Mori Y, Peskin C (2009) A numerical method for cellular electrophysiology based on the electrodiffusion equations with internal boundary conditions at internal membranes. Commun Appl Math Comput Sci 4:85-134

Mori Y, Liu C, Eisenberg R (2011) A model of electrodiffusion and osmotic water flow and its energetic structure. Phys D Nonlinear Phenom 240:1835-1852

Onsager L (1931) Reciprocal relations in irreversible processes II. Phys Rev 38(12):2265-2279

Sauer F (1973) Nonequilibrium thermodynamics of kidney tubule transport. In: Orloff J, Berliner R (eds) Handbook of physiology, section 8, renal physiology. American Physiological Society, USA pp 399414

Strieter J, Stephenson JL, Palmer LG, Weinstein AM (1990) Volume-activated chloride permeability can mediate cell volume regulation in a mathematical model of a tight epithelium. J Gen Physiol 96(2):319 
Tosteson D (1964) Regulation of cell volume by sodium and potassium transport. The cellular functions of membrane transport. Prentice Hall, Englewood Cliffs, pp 3-22

Tosteson D, Hoffman J (1960) Regulation of cell volume by active cation transport in high and low potassium sheep red cells. J Gen Physiol 44(1):169

Weinstein A (1983) Nonequilibrium thermodynamic model of the rat proximal tubule epithelium. Biophys J 44(2):153-170

Weinstein A (1992) Analysis of volume regulation in an epithelial cell model. Bull Math Biol 54(4): 537-561

Weinstein A (1994) Mathematical models of tubular transport. Annu Rev Physiol 56(1):691-709

Weinstein A (1997) Dynamics of cellular homeostasis: recovery time for a perturbation from equilibrium. Bull Math Biol 59(3):451-481

Weinstein A (2002) Assessing homeostatic properties of epithelial cell models: application to kidney proximal tubule. In: Layton H, Weinstein A (eds) Membrane transport and renal physiology. IMA volumes in mathematics and its applications, vol 129. Springer, Berlin pp 119-140

Weinstein A (2003) Mathematical models of renal fluid and electrolyte transport: acknowledging our uncertainty. Am J Physiol Renal Physiol 284(5):871

Weinstein A (2004) Modeling epithelial cell homeostasis: assessing recovery and control mechanisms. Bull Math Biol 66(5):1201-1240

Weinstein A, Sontag E (2009) Modeling proximal tubule cell homeostasis: tracking changes in luminal flow. Bull Math Biol 71(6):1285-1322

Yi C, Fogelson A, Keener J, Peskin C (2003) A mathematical study of volume shifts and ionic concentration changes during ischemia and hypoxia. J Theor Biol 220(1):83-106 